\title{
SOLVABILITY OF A VOLUME INTEGRAL EQUATION FORMULATION FOR ANISOTROPIC ELASTODYNAMIC SCATTERING
}

\author{
MARC BONNET
}

\author{
Communicated by Fioralba Cakoni
}

\begin{abstract}
This article investigates the solvability of volume integral equations arising in elastodynamic scattering by penetrable obstacles. The elasticity tensor and mass density are allowed to be smoothly heterogeneous inside the obstacle and may be discontinuous across the backgroundobstacle interface, the background elastic material being homogeneous. Both materials may be anisotropic, within certain limitations for the background medium. The volume integral equation associated with this problem is first derived, relying on known properties of the background fundamental tensor. To avoid difficulties associated with existing radiation conditions for anisotropic elastic media, we also propose a definition of the radiating character of transmission solutions. The unique solvability of the volume integral equation (and of the scattering problem) is established. For the important special case of isotropic background properties, our definition of a radiating solution is found to be equivalent to the Sommerfeld-Kupradze radiation conditions. Moreover, solvability for anisotropic elastostatics, directly related to known results on the equivalent inclusion method, is recovered as a by-product.
\end{abstract}

1. Introduction. Volume integral equations, also known as Lippmann-Schwinger integral equations, arise naturally when considering the scattering of waves by penetrable inhomogeneities embedded in a homogeneous background medium, for which a fundamental solution is known. They have been developed and used in various areas of physics, such as electromagnetism and optics [3, Chapter 2], [8, Chapter 9],

2010 AMS Mathematics subject classification. Primary 35J15, 45F15, 65R20, $74 \mathrm{~J} 20$.

Keywords and phrases. Volume integral equation, elastodynamics, anisotropy, scattering.

Received by the editors on July 27, 2015, and in revised form on December 2, 2015. 
acoustics [9, Chapter 8], [28], or elastodynamics [34, 35, 39], for a long time. If the penetrable object has homogeneous properties, scattering may alternatively be modeled using coupled surface integral equations, see e.g., [13] for elastodynamics. Variational formulations, combined with appropriate handling of the solution behavior at infinity, can also be applied to such scattering problems.

Volume integral equations have a geometrical support restricted to the spatial region where material properties differ from the background. This feature makes them useful, e.g., for deriving asymptotic or homogenized models involving inhomogeneities of low contrast or vanishing size $[\mathbf{2}, \mathbf{3 8}]$. Moreover, as they provide a direct mathematical link between unknown inhomogeneities and remote measurements, they are also convenient for medium imaging inverse problems [5], for instance, providing a foundation for contrast source inversion methods [32] or allowing rather explicit expressions of far-field patterns.

In contrast with the vast existing literature on the mathematical aspects of boundary integral equations and their application to scattering by impenetrable obstacles characterized by Dirichlet, Neumann or impedant boundary conditions, comparatively few studies are available regarding the mathematical properties of volume integral equations. The well-posedness of volume integral formulations for various electromagnetic scattering problems is addressed in [33] for twodimensional orthotropic inhomogeneities and in $[\mathbf{2 1}, \mathbf{2 2}, \mathbf{2 3}, \mathbf{2 4}]$ for three-dimensional problems where connections to (and complementarity with) variational formulations are emphasized. Solvability and other mathematical properties of volume integral equations for threedimensional electromagnetic scattering are addressed in $[\mathbf{1 0}, \mathbf{1 1}]$. All of these studies assume isotropic properties for the background material.

The goal of this article is to establish the solvability of volume integral equations associated with elastodynamic scattering by penetrable obstacles, a question which to the best of our knowledge is not addressed in the available literature (see, however, [18] for obstacles characterized by a mass density perturbation and [16] for Eshelby-type elastostatic problems involving elastic inhomogeneities). The relevant material properties (elasticity tensor, mass density) are assumed to be smooth inside the obstacle; they may be discontinuous across the background-obstacle interface and are otherwise allowed to be arbitrary (e.g., in terms of heterogeneity or elastic anisotropy). The background 
elastic material is assumed to be homogeneous. To make this study as general as possible, anisotropy is also allowed for the background material. The elastodynamic fundamental tensor for the background medium is then known as a Fourier integral that, unlike in the isotropic case, cannot be evaluated in closed form. The background anisotropy permitted is constrained by a set of assumptions, expressed in terms of the geometry of the slowness surfaces, that allow reasonably simple far-field asymptotic formulas for the fundamental tensor, a framework that was previously used in [31] for studying the solvability of surface integral formulations of anisotropic elastodynamic exterior problems. We thus rely on some results of [31] regarding, e.g., properties of the anisotropic fundamental tensor. The subclass of anisotropic background permitted properties, termed as class I anisotropy in the threeclass categorization of [4], includes isotropic materials as an important special case.

The assumption of background anisotropy complicates the solvability problem at hand. First, the available generalization of the Sommerfeld-Kupradze radiation conditions $[31,36]$ is inconvenient, prompting us to adopt instead (inspired by [12] and [29, Chapter 7]) another definition of the radiating character of transmission solutions that is easier to formulate and use, and is equivalent to Sommerfeldtype radiation conditions in situations when the latter are available. We also show that this definition achieves solutions that have outgoing energy flux, i.e., are radiating in a physical sense. Moreover, the unavailability of expansions of elastodynamic solutions as a series of eigenfunctions deprives one of Rellich's lemma, so we rely instead on a theorem [26] valid for a large class of linear partial differential operators, which, however, must also here conform to the class I anisotropy restrictions.

This article is organized as follows. The rest of this section is devoted to introducing the scattering problem of interest and collecting background and notation pertaining to anisotropic elastodynamics. In Section 2, we derive the governing volume integral equation for this problem, relying on known properties of the background fundamental tensor that are recalled along the way, and specifying a definition of the radiating character of transmission solutions which is both appropriate and convenient for the context of this work. The unique solvability of the volume integral equation (and of the scattering problem), which is 
our main result, is established in Section 3. Remarks on the important special case of isotropic background properties are given in Section 4, where our definition of a radiating solution is in particular shown to be equivalent to the Sommerfeld-Kupradze radiation conditions [25]. Finally, unique solvability for anisotropic elastostatics is obtained as a by-product in Section 5, this time without restrictions on anisotropy; this result is closely related to that of [16] on the equivalent-inclusion method. Some auxiliary proofs are finally given in Section 6 .

1.1. Formulation of the problem. We consider the scattering of time-harmonic elastic waves by an elastic inhomogeneity embedded in an unbounded background elastic medium. The inhomogeneity occupies a bounded domain $D_{1} \subset \mathbb{R}^{3}$. The unbounded complement $D_{0}:=\mathbb{R}^{3} \backslash \overline{D_{1}}$ of $D_{1}$ is assumed to be connected, the background material filling $D_{0}$ being homogeneous and anisotropic. The constitutive material of the scatterer is also anisotropic and may be heterogeneous. The (possibly anisotropic) elastic properties of the background medium and the scatterer are, respectively, characterized by the fourth-order elasticity tensors $\mathcal{C}^{0}$ and $\mathcal{C}^{1}$. The corresponding mass densities are $\rho^{0}$ and $\rho^{1}$. These material characteristics are all real-valued. Moreover, $\mathcal{C}^{0}$ and $\rho^{0}$ are assumed to be uniform, while $\mathcal{C}^{1}$ and $\rho^{1}$ are $C^{1, \alpha}\left(\overline{D_{1}}\right)$ functions. The primary field variable characterizing an elastodynamic state is the vector-valued displacement. For a given displacement field $\boldsymbol{w}$, the stress tensor $\boldsymbol{\sigma}_{\ell}[\boldsymbol{w}]$ in $D_{\ell}(\ell=0,1)$ is then given by $\boldsymbol{\sigma}_{\ell}[\boldsymbol{w}]=\mathcal{C}^{\ell}: \varepsilon[\boldsymbol{w}]$, where $\boldsymbol{\varepsilon}[\boldsymbol{w}]:=\frac{1}{2}\left(\boldsymbol{\nabla} \boldsymbol{w}+\boldsymbol{\nabla} \boldsymbol{w}^{\mathrm{T}}\right)$ is the linearized strain tensor associated with $\boldsymbol{w}$. The tractions $\boldsymbol{t}_{\ell}[\boldsymbol{w}]$, i.e., the force surface densities exerted by medium $\ell$ on the interface $\Gamma$ separating $D_{0}$ and $D_{1}$ (with $\boldsymbol{n}$ conventionally denoting the unit normal to $\Gamma$ pointing outwards of $D_{1}$ ), are then defined by

$$
\boldsymbol{t}_{\ell}[\boldsymbol{w}](\boldsymbol{x})=\lim _{h>0 \rightarrow 0} \boldsymbol{\sigma}_{\ell}[\boldsymbol{w}]\left(\boldsymbol{x}+(-1)^{\ell} h \boldsymbol{n}\right) \cdot \boldsymbol{n}(\boldsymbol{x}), \quad \boldsymbol{x} \in \Gamma, \ell=0,1 .
$$

In (1.1) and hereinafter, the symbols '.' and ': ' denote single and double inner products, e.g., $(\boldsymbol{\sigma} \cdot \boldsymbol{n})_{i}=\sigma_{i j} n_{j}$ and $\left(\mathcal{C}^{0}: \boldsymbol{\varepsilon}\right)_{i j}=\mathcal{C}_{i j k \ell}^{0} \varepsilon_{k \ell}$, with Einstein's convention of summation over repeated indices implicitly used throughout and component indices always referring to an orthonormal frame. To define a physically meaningful problem, the material parameters obey the following assumptions: the elasticity tensors $\mathcal{C}^{\ell}$ verify $\mathcal{C}_{i j k \ell}^{\ell}=\mathcal{C}_{k \ell i j}^{\ell}=\mathcal{C}_{i j \ell k}^{\ell}$ (major and minor symmetries) and define 
symmetric positive quadratic forms over the symmetric second-order tensors (i.e., $\varepsilon: \mathcal{C}^{\ell}: \varepsilon>0$ for any $\varepsilon \in \mathbb{R}_{\text {sym }}^{3 \times 3} \backslash\{\mathbf{0}\}$ ), while the mass densities $\rho^{\ell}$ are strictly positive and bounded away from zero.

In the absence of body forces, any displacement field $\boldsymbol{w}$ in $D_{\ell}$ $(\ell=0,1)$ satisfies the homogeneous time-harmonic elastodynamic field equation

$$
\mathcal{B}_{\ell} \boldsymbol{w}=\mathbf{0} \text { in } D_{\ell}
$$

where the partial differential operator $\boldsymbol{B}_{\ell}$ associated to medium $\ell$, defined by

$$
\mathcal{B}_{\ell} \boldsymbol{w}=-\operatorname{div}\left(\mathcal{C}^{\ell}: \varepsilon[\boldsymbol{w}]\right)-\rho^{\ell} \omega^{2} \boldsymbol{w}
$$

(with the divergence of any second-order tensor field $\boldsymbol{\sigma}$ defined by $\left.(\operatorname{div} \boldsymbol{\sigma})_{i}=\partial_{j} \sigma_{i j}\right)$, is strongly elliptic [29, Chapter 4] and formally self-adjoint. The time-harmonic factor $e^{-\mathrm{i} \omega t}$ is implicitly understood for all field quantities.

The transmission problem under consideration concerns the scattering by the inhomogeneity of a given incident field $\boldsymbol{u}_{\mathrm{I}}$, which is an elastodynamic solution for the background medium, i.e., it satisfies

$$
\mathcal{B}_{0} \boldsymbol{u}_{\mathrm{I}}=\mathbf{0} \text { in } \mathbb{R}^{3}
$$

Writing the total displacement field $\boldsymbol{u}$ in $D_{0} \cup D_{1}$ in the form $\boldsymbol{u}=\boldsymbol{v}+\boldsymbol{u}_{\mathrm{I}}$, the restrictions $\boldsymbol{v}_{0}:=\left.\boldsymbol{v}\right|_{D_{0}}$ and $\boldsymbol{v}_{1}:=\left.\boldsymbol{v}\right|_{D_{1}}$ of the scattered field $\boldsymbol{v}$ satisfy the field equations

$$
\text { (a) } \mathcal{B}_{0}\left(\boldsymbol{v}_{0}+\boldsymbol{u}_{\mathrm{I}}\right)=\mathbf{0} \text { in } D_{0} \text {, (b) } \mathcal{B}_{1}\left(\boldsymbol{v}_{1}+\boldsymbol{u}_{\mathrm{I}}\right)=\mathbf{0} \text { in } D_{1} \text {, }
$$

and the transmission conditions

$$
\text { (a) } \boldsymbol{v}_{1}=\boldsymbol{v}_{0}, \quad \text { (b) } \boldsymbol{t}_{1}\left[\boldsymbol{v}_{1}+\boldsymbol{u}_{\mathrm{I}}\right]=\boldsymbol{t}_{0}\left[\boldsymbol{v}_{0}+\boldsymbol{u}_{\mathrm{I}}\right] \text { on } \Gamma \text {. }
$$

In what follows, any solution $\boldsymbol{v}$ to (1.5) and (1.6) will be called a transmission solution.

In addition, $\boldsymbol{v}_{0}$ is assumed to be radiating at infinity. Radiation conditions for anisotropic elastic media, while known [36], are cumbersome as they involve an additive split of $\boldsymbol{v}_{0}$ into three parts that are not explicitly defined. An alternative, simpler, definition for the radiating character of $\boldsymbol{v}_{0}$ will be stated and adopted later in this article 
(see Definition 2.6 in subsection 2.3); until then, the precise meaning of "radiating" is left unspecified.

We are interested in transmission solutions $\boldsymbol{v}$ which have finite local energy: $\boldsymbol{v} \in \boldsymbol{H}_{\mathrm{loc}}^{1}\left(\mathbb{R}^{3}\right)$, i.e., $\boldsymbol{v}_{1} \in \boldsymbol{H}^{1}\left(D_{1}\right)$ and $\boldsymbol{v}_{0} \in \boldsymbol{H}_{\mathrm{loc}}^{1}\left(D_{0}\right)$, where boldface symbols $\boldsymbol{H}^{1}, \boldsymbol{L}^{2} \ldots$, indicate Sobolev function spaces of complex-valued vector fields, e.g., $\boldsymbol{H}^{1}(X):=H^{1}\left(X ; \mathbb{C}^{3}\right)$ for some domain $X \subset \mathbb{R}^{3}$. We introduce the bilinear forms

$$
\begin{aligned}
\langle\boldsymbol{u}, \boldsymbol{w}\rangle_{X}^{\mathcal{C}} & :=\int_{X} \varepsilon[\boldsymbol{u}]: \mathcal{C}: \varepsilon[\boldsymbol{w}] \mathrm{d} V=\int_{X} \boldsymbol{\nabla} \boldsymbol{u}: \mathcal{C}: \nabla \boldsymbol{w} \mathrm{d} V \\
(\boldsymbol{u}, \boldsymbol{w})_{X}^{\rho} & :=\int_{X} \rho \boldsymbol{u} \cdot \boldsymbol{w} \mathrm{d} V
\end{aligned}
$$

respectively associated with elastic strain energy and kinetic energy, for some domain $X \subset \mathbb{R}^{3}$, elasticity tensor $\mathcal{C}$ and mass density $\rho$. Any transmission solution $\boldsymbol{v} \in \boldsymbol{H}_{\mathrm{loc}}^{1}\left(\mathbb{R}^{3}\right)$ of (1.5) and (1.6) then satisfies

$$
\langle\boldsymbol{v}, \boldsymbol{\psi}\rangle_{\mathbb{R}^{3}}^{\mathcal{C}}-\omega^{2}(\boldsymbol{v}, \boldsymbol{\psi})_{\mathbb{R}^{3}}^{\rho}=-\left\langle\boldsymbol{u}_{\mathrm{I}}, \boldsymbol{\psi}\right\rangle_{D_{1}}^{\Delta \mathcal{C}}+\omega^{2}\left(\boldsymbol{u}_{\mathrm{I}}, \boldsymbol{\psi}\right)_{D_{1}}^{\Delta \rho},
$$

for all test displacements $\boldsymbol{\psi} \in \boldsymbol{H}_{\text {comp }}^{1}\left(\mathbb{R}^{3}\right)$, having set $\mathcal{C}:=\mathcal{C}^{0}+$ $(\Delta \mathcal{C}) 1_{D_{1}}$ and $\rho:=\rho^{0}+(\Delta \rho) 1_{D_{1}}$; this stems from taking the inner product of (1.4) and (1.5) (a), (b) with $\boldsymbol{\psi} \in \boldsymbol{H}_{\text {comp }}^{1}\left(\mathbb{R}^{3}\right)$, invoking in each case the first Green identity, and combining the resulting equalities. Equation (1.9) is the variational form of

$$
\mathcal{B} \boldsymbol{v}=\operatorname{div}\left(\Delta \mathcal{C}: \varepsilon\left[\boldsymbol{u}_{\mathrm{I}}\right]\right)+\Delta \rho \omega^{2} \boldsymbol{u}_{\mathrm{I}} \quad \text { in } \mathbb{R}^{3} \backslash \Gamma
$$

(with $\left.\mathcal{B} \boldsymbol{v}:=-\operatorname{div}(\mathcal{C}: \varepsilon[\boldsymbol{v}])-\rho \omega^{2} \boldsymbol{v}\right)$ combined with the transmission conditions (1.6).

1.2. Auxiliary results and notation. This section collects auxiliary results and notation associated with the framework of [31], adopted in this study, regarding anisotropic elastodynamics and constraints (stated in Assumption 1.1 below) on the background elasticity tensor.

1.2.1. Matrix form of elastodynamic equation. The general inhomogeneous elastodynamic equation $\boldsymbol{B}_{0} \boldsymbol{w}=\boldsymbol{f}$ for the background medium can be given the equivalent matrix form

$$
\boldsymbol{B}_{0}(-\mathrm{i} \boldsymbol{\nabla}) \boldsymbol{w}=\boldsymbol{f}
$$


with $\boldsymbol{\nabla}=\left(\partial_{1}, \partial_{2}, \partial_{3}\right), \boldsymbol{B}_{0}(\boldsymbol{\xi})=\boldsymbol{A}(\boldsymbol{\xi})-\rho^{0} \omega^{2} \boldsymbol{I}$ and the acoustic tensor $\boldsymbol{A}(\boldsymbol{\xi})$ defined by $A_{i k}(\boldsymbol{\xi})=\mathcal{C}_{i j k \ell}^{0} \xi_{j} \xi_{\ell}$. The positive definiteness of $\varepsilon \mapsto \varepsilon: \mathcal{C}^{0}: \varepsilon$ over $\mathbb{R}_{\mathrm{sym}}^{3 \times 3}$ implies that $\boldsymbol{A}(\boldsymbol{\xi}) \in \mathbb{R}^{3 \times 3}$ is positive definite for any $\boldsymbol{\xi} \in \mathbb{R}^{3} \backslash\{\mathbf{0}\}$. Let

$$
D(\boldsymbol{\xi})=\operatorname{Det}\left(\boldsymbol{B}_{0}(\boldsymbol{\xi})\right)=\operatorname{Det}\left(\boldsymbol{A}(\boldsymbol{\xi})-\rho^{0} \omega^{2} \boldsymbol{I}\right)
$$

denote the characteristic determinant of $\boldsymbol{B}_{0}$. The adjugate matrix $\boldsymbol{N}(\boldsymbol{\xi})$ of $\boldsymbol{B}_{0}(\boldsymbol{\xi})$, i.e., the transpose of its cofactor matrix, is then given (since $\boldsymbol{B}_{0}(\boldsymbol{\xi}) \in \mathbb{R}^{3 \times 3}$ ) by

$$
\boldsymbol{N}(\boldsymbol{\xi})=\left(\frac{1}{2}\left(\left[\operatorname{Tr}\left(\boldsymbol{B}_{0}\right)\right]^{2}-\operatorname{Tr}\left(\boldsymbol{B}_{0}^{2}\right)\right) \boldsymbol{I}-\operatorname{Tr}\left(\boldsymbol{B}_{0}\right) \boldsymbol{B}_{0}+\boldsymbol{B}_{0}^{2}\right)(\boldsymbol{\xi}),
$$

and is such that

$$
\boldsymbol{B}_{0}(\boldsymbol{\xi}) \cdot \boldsymbol{N}(\boldsymbol{\xi})=\boldsymbol{N}(\boldsymbol{\xi}) \cdot \boldsymbol{B}_{0}(\boldsymbol{\xi})=D(\boldsymbol{\xi}) \boldsymbol{I} .
$$

In particular, when $\boldsymbol{B}_{0}(\boldsymbol{\xi})$ is invertible, $\boldsymbol{B}_{0}{ }^{-1}(\boldsymbol{\xi})=D(\boldsymbol{\xi})^{-1} \boldsymbol{N}(\boldsymbol{\xi})$. Finally, defining $\widehat{\boldsymbol{A}}(\boldsymbol{n}, \boldsymbol{\xi})$ by $\widehat{A}_{i k}(\boldsymbol{n}, \boldsymbol{\xi})=\mathcal{C}_{i j k \ell}^{0} n_{j} \xi_{\ell}, 1 \leq i, k \leq 3$, the traction operator $\boldsymbol{w} \mapsto \boldsymbol{t}_{0}[\boldsymbol{w}]$ can be given the form

$$
\boldsymbol{t}_{0}[\boldsymbol{w}]=\widehat{A}(\boldsymbol{n}, \boldsymbol{\nabla}) \boldsymbol{w} .
$$

1.2.2. Characteristic (slowness) surfaces. Plane waves, i.e, displacement fields $\boldsymbol{w}$ of the form $\boldsymbol{w}(\boldsymbol{x})=e^{\mathrm{i} \boldsymbol{\xi} \cdot \boldsymbol{x}} \boldsymbol{b}$ in the background medium (with the vectors $\boldsymbol{\xi}, \boldsymbol{b} \in \mathbb{R}^{3}$ defining propagation and polarization directions, respectively), solve the field equation $\boldsymbol{B}_{0}(-\mathrm{i} \boldsymbol{\nabla}) \boldsymbol{w}=\mathbf{0}$, provided the linear system $\boldsymbol{B}_{0}(\boldsymbol{\xi}) \cdot \boldsymbol{b}=\mathbf{0}$ has nonzero solutions $\boldsymbol{b}=\boldsymbol{b}(\boldsymbol{\xi})$. The propagation vector $\boldsymbol{\xi}$ must therefore satisfy the characteristic equation

$$
D(\boldsymbol{\xi})=0 .
$$

Setting $\boldsymbol{\xi}=\lambda \widehat{\boldsymbol{\xi}}$ with $\widehat{\boldsymbol{\xi}} \in \widehat{S}$ and $\lambda>0(\widehat{S}$ denoting the unit sphere in $\mathbb{R}^{3}$ ), the above characteristic equation implies, for given propagation direction $\widehat{\boldsymbol{\xi}} \in \widehat{S}$, that $\rho^{0} \omega^{2} / \lambda^{2}$ are eigenvalues of the (real, symmetric, positive definite) matrix $\boldsymbol{A}(\widehat{\boldsymbol{\xi}})$. Counting possible multiplicities, there are three such values $0<\alpha^{1}(\widehat{\boldsymbol{\xi}}) \leq \alpha^{2}(\widehat{\boldsymbol{\xi}}) \leq \alpha^{3}(\widehat{\boldsymbol{\xi}})$. The corresponding polarization directions $\boldsymbol{b}^{q}$ (where $\left|\boldsymbol{b}^{q}\right|=1$ may be assumed) solve $\boldsymbol{B}_{0}\left(\lambda^{q} \widehat{\boldsymbol{\xi}}\right) \cdot \boldsymbol{b}^{q}(\widehat{\boldsymbol{\xi}})=\mathbf{0}$, and $\boldsymbol{B}_{0}(\boldsymbol{\xi})$ is given in terms of the $\alpha^{q}$ and $\boldsymbol{b}^{q}$ 
by

$$
\boldsymbol{B}_{0}(\boldsymbol{\xi})=\sum_{q=1}^{3}\left(|\boldsymbol{\xi}|^{2} \alpha^{q}(\widehat{\boldsymbol{\xi}})-\rho^{0} \omega^{2}\right) \boldsymbol{b}^{q}(\widehat{\boldsymbol{\xi}}) \otimes \boldsymbol{b}^{q}(\widehat{\boldsymbol{\xi}})
$$

Real solutions of $D(\boldsymbol{\xi})=0$ form three characteristic surfaces $S^{q}, q=$ $1,2,3$, in $\boldsymbol{\xi}$-space, defined by $S^{q}=\left\{\boldsymbol{\xi} \in \mathbb{R}^{3}, \boldsymbol{\xi}=\left[\rho^{0} \omega^{2} / \alpha^{q}(\widehat{\boldsymbol{\xi}})\right] \widehat{\boldsymbol{\xi}}, \widehat{\boldsymbol{\xi}} \in \widehat{S}\right\}$. The $S^{q}$ are often called slowness surfaces [4]. The adjugate matrix $\boldsymbol{N}(\boldsymbol{\xi})$ of $\boldsymbol{B}_{0}(\boldsymbol{\xi})$ for $\boldsymbol{\xi} \in S^{q}$ has rank 1 and is given, when the $S^{q}$ are all distinct, by

$$
\begin{aligned}
& \boldsymbol{N}(\boldsymbol{\xi})=\gamma^{q}(\widehat{\boldsymbol{\xi}}) \boldsymbol{b}^{q}(\widehat{\boldsymbol{\xi}}) \otimes \boldsymbol{b}^{q}(\widehat{\boldsymbol{\xi}}), \\
& \gamma^{q}(\widehat{\boldsymbol{\xi}})=\left(\rho^{0} \omega^{2}\right)^{2} \prod_{p \neq q}\left(1-\alpha^{p}(\widehat{\boldsymbol{\xi}}) / \alpha^{q}(\widehat{\boldsymbol{\xi}})\right), \quad \boldsymbol{\xi} \in S^{q} .
\end{aligned}
$$

In particular, we have $(-1)^{q} \gamma^{q}(\widehat{\boldsymbol{\xi}})>0, q=1,2,3$.

1.2.3. Restrictions on background anisotropy. For reasons which will appear later, the permitted anisotropic elastic properties of the background material are subject to the following constraints:

Assumption 1.1. The background elasticity tensor $\mathcal{C}^{0}$ is such that the following conditions (which define the class I anisotropy in the threeclass categorization of [4]) are satisfied:

(i) $\boldsymbol{\nabla} D \neq \mathbf{0}$ at any real zero of the polynomial $D(\boldsymbol{\xi})$;

(ii) The Gaussian curvature $\kappa^{q}$ of $S^{q}$ does not vanish anywhere on $S^{q}, q=1,2,3$.

Assumption 1.1 ensures that $S^{q}$ are closed, convex and non-selfintersecting surfaces containing the origin in their interior [31]. The outward unit normal to $S^{q}$ being given by $\boldsymbol{\nu}(\boldsymbol{\xi})=(-1)^{q} \boldsymbol{\nabla} D(\boldsymbol{\xi}) /|\boldsymbol{\nabla} D(\boldsymbol{\xi})|$, conditions (i) and (ii) also imply that, for any given unit direction $\widehat{\boldsymbol{x}} \in \widehat{S}$, there exists a unique vector $\boldsymbol{\xi}^{q}(\widehat{\boldsymbol{x}})$ on $S^{q}$ such that $\boldsymbol{\nu}\left(\boldsymbol{\xi}^{q}\right)=\widehat{\boldsymbol{x}}$ (in which case we also have $-\boldsymbol{\xi}^{q} \in S^{q}$ and $\boldsymbol{\nu}\left(-\boldsymbol{\xi}^{q}\right)=-\widehat{\boldsymbol{x}}$ ). 


\section{Volume integral equation of Lippmann-Schwinger type.}

2.1. Elastodynamic radiating fundamental tensor. Let $G=$ $\boldsymbol{e}_{k} \otimes \boldsymbol{G}^{k}$ be the fundamental tensor for the unbounded background medium, with each vector $G^{k}$ defined as the radiating displacement field solving

$$
\mathcal{B}_{0} \boldsymbol{G}^{k}=\delta \boldsymbol{e}_{k}
$$

( $\delta$ denoting the Dirac distribution supported at the origin). As shown in [31], $\boldsymbol{G}$ is given by

$$
\begin{aligned}
\boldsymbol{G}(\boldsymbol{x})=\lim _{\epsilon>0 \rightarrow 0} & \lim _{R \rightarrow+\infty} \frac{1}{(2 \pi)^{3}} \\
& \times \int_{|\boldsymbol{\xi}| \leq R}\left[\boldsymbol{A}(\boldsymbol{\xi})-\rho^{0}(\omega+\mathrm{i} \epsilon)^{2} \boldsymbol{I}\right]^{-1} e^{\mathrm{i} \boldsymbol{\xi} \cdot \boldsymbol{x}} \mathrm{d} V(\boldsymbol{\xi}),
\end{aligned}
$$

with the chosen limiting process with respect to $\epsilon$ expressing the limiting absorption principle. No closed-form expression of (2.2) is known for general anisotropic elasticity (whereas one is available for isotropic elasticity, see e.g., $[\mathbf{1 4}, \mathbf{2 5}]$ and Section 4$). \boldsymbol{G}(\boldsymbol{x})$ has the properties

$$
\begin{array}{lll}
\text { (a) } \boldsymbol{G}(\boldsymbol{x})=\boldsymbol{G}(-\boldsymbol{x})=\boldsymbol{G}^{\mathrm{T}}(\boldsymbol{x}) & & \text { (symmetry), } \\
\text { (b) } \boldsymbol{G}(\boldsymbol{x})=O\left(|\boldsymbol{x}|^{-1}\right),|\boldsymbol{x}| \rightarrow 0 & & \text { (singularity), }
\end{array}
$$

which can be proved by a suitable change of variables in the Fourier integral representation (2.2) (or inferred from the alternative representation of $\boldsymbol{G}$ given in [37], based on the Radon transform). Moreover, translational invariance of the homogeneous full space implies that the fundamental tensor generated by a point source located at $\boldsymbol{y}$ is $\boldsymbol{G}(\boldsymbol{x}-\boldsymbol{y})$.

The far-field asymptotic behavior of $\boldsymbol{G}(\boldsymbol{x}-\boldsymbol{y})$ for $|\boldsymbol{x}| \rightarrow \infty$ (with $\boldsymbol{y}$ fixed) is given by

$$
\boldsymbol{G}(\boldsymbol{x}-\boldsymbol{y})=\left(\sum_{q=1}^{3}(-1)^{q} E\left(\boldsymbol{\xi}^{q}\right) \boldsymbol{N}\left(\boldsymbol{\xi}^{q}\right) e^{\mathrm{i} \boldsymbol{\xi}^{q} \cdot(\boldsymbol{x}-\boldsymbol{y})}\right)|\boldsymbol{x}|^{-1}+O\left(|\boldsymbol{x}|^{-2}\right)
$$

(see [31, Lemma 3.2]) with $\boldsymbol{\xi}^{q}=\boldsymbol{\xi}^{q}(\widehat{\boldsymbol{x}})$ and

$$
E\left(\boldsymbol{\xi}^{q}\right)=\left(2 \pi\left|\nabla D\left(\boldsymbol{\xi}^{q}\right)\right|\right)^{-1}\left[\kappa\left(\boldsymbol{\xi}^{q}\right)\right]^{-1 / 2} .
$$


Using (1.13), the corresponding far-field asymptotic form of the fundamental traction tensor $\boldsymbol{T}=\boldsymbol{e}_{k} \otimes \boldsymbol{T}^{k}$ such that $\boldsymbol{T}^{k}:=\boldsymbol{t}_{0}\left[\boldsymbol{G}^{k}\right]$ is then

$$
\begin{aligned}
\boldsymbol{T}(\boldsymbol{x}-\boldsymbol{y})= & \left(\mathrm{i} \sum_{q=1}^{3}(-1)^{q} E\left(\boldsymbol{\xi}^{q}\right) \widehat{\boldsymbol{A}}\left(\boldsymbol{n}(\boldsymbol{x}), \boldsymbol{\xi}^{q}\right) \cdot \boldsymbol{N}\left(\boldsymbol{\xi}^{q}\right) e^{\mathrm{i} \boldsymbol{\xi}^{q} \cdot(\boldsymbol{x}-\boldsymbol{y})}\right)|\boldsymbol{x}|^{-1} \\
& +O\left(|\boldsymbol{x}|^{-2}\right) .
\end{aligned}
$$

Remark 2.1. The far-field asymptotic formulas (2.4a), (2.4b) are valid for anisotropic materials satisfying Assumption 1.1 [31], so that $\boldsymbol{\xi}^{q}(\widehat{\boldsymbol{x}})$ are the (nondegenerate) critical points arising in a stationaryphase approximation of the integral representation (2.2) for $|\boldsymbol{x}| \rightarrow \infty$. Materials of this class for which the characteristic surfaces $S^{q}$ are in addition ellipsoidal are determined in [6]. Anisotropic elasticity tensors failing to satisfy Assumption 1.1, i.e., corresponding to class II or III materials in [4], give rise to additional far-field contributions, some of them decaying at a rate slower than $O\left(|\boldsymbol{x}|^{-1}\right)$ along certain observation directions $\widehat{\boldsymbol{x}}$ (see, e.g., [17]), making the far-field behavior of $\boldsymbol{G}$ significantly more complex; they are not considered in this work.

2.2. Volume potentials. Define the volume vector potentials $\mathcal{V}_{\omega}$ and $\mathcal{W}_{\omega}$, for respective densities $\boldsymbol{g} \in \boldsymbol{L}^{2}\left(D_{1}\right)$ and $\boldsymbol{h} \in L^{2}\left(D_{1} ; \mathbb{C}^{3 \times 3}\right)$, by

$$
\begin{aligned}
\mathcal{V}_{\omega}[\boldsymbol{g}](\boldsymbol{x}) & =\int_{D_{1}} \boldsymbol{G}(\boldsymbol{x}-\boldsymbol{y}) \cdot \boldsymbol{g}(\boldsymbol{y}) \mathrm{d} V(\boldsymbol{y}), \\
\mathcal{W}_{\omega}[\boldsymbol{h}](\boldsymbol{x}) & =\operatorname{div} \int_{D_{1}} \boldsymbol{G}(\boldsymbol{x}-\boldsymbol{y}) \cdot \boldsymbol{h}(\boldsymbol{y}) \mathrm{d} V(\boldsymbol{y}),
\end{aligned}
$$

with the divergence operator in (2.5b) defined as in (1.3). By virtue of known mapping properties of integral operators treated as pseudodifferential operators [20, Theorem 6.1.12], $\mathcal{V}_{\omega}$ and $\mathcal{W}_{\omega}$ are well defined as $L^{2}\left(D_{1} ; \mathbb{C}^{3}\right) \rightarrow \boldsymbol{H}_{\text {loc }}^{2}\left(\mathbb{R}^{3}\right)$ and $L^{2}\left(D_{1} ; \mathbb{C}^{3 \times 3}\right) \rightarrow \boldsymbol{H}_{\text {loc }}^{1}\left(\mathbb{R}^{3}\right)$ operators, respectively. Moreover, we note for later reference that $\mathcal{W}_{\boldsymbol{\omega}}[\boldsymbol{h}]$ can be reformulated by using property (2.3a) of $\boldsymbol{G}$, to obtain

$$
\mathcal{W}_{\omega}[\boldsymbol{h}](\boldsymbol{x})=\int_{D_{1}} \boldsymbol{\nabla} \boldsymbol{G}(\boldsymbol{x}-\boldsymbol{y}): \boldsymbol{h}(\boldsymbol{y}) \mathrm{d} S_{y}
$$




$$
\begin{aligned}
& =-\int_{D_{1}} \boldsymbol{\nabla} \boldsymbol{G}(\boldsymbol{y}-\boldsymbol{x}): \boldsymbol{h}(\boldsymbol{y}) \mathrm{d} S_{y} \\
& =\mathcal{V}_{\omega}[\operatorname{div} \boldsymbol{h}](\boldsymbol{x})-\mathcal{S}_{\omega}[\boldsymbol{h} \cdot \boldsymbol{n}](\boldsymbol{x}),
\end{aligned}
$$

where the last equality results from an integration by parts and

$$
\mathcal{S}_{\omega}[\boldsymbol{f}](\boldsymbol{x}):=\int_{\Gamma} \boldsymbol{G}(\boldsymbol{y}-\boldsymbol{x}) \cdot \boldsymbol{f}(\boldsymbol{y}) \mathrm{d} S_{y}
$$

is the elastodynamic single-layer potential with density $\boldsymbol{f}$, satisfying $\mathcal{B}_{0} \mathcal{S}_{\omega}[\boldsymbol{f}]=\mathbf{0}$ in $\mathbb{R}^{3} \backslash \Gamma$.

The following two lemmas state properties of fields given by volume potentials that will be useful later in proving uniqueness for the transmission problem.

Lemma 2.2. Any displacement field $\boldsymbol{w}$ of the form $\boldsymbol{w}=\mathcal{V}_{\omega}[\boldsymbol{g}]+\mathcal{W}_{\omega}[\boldsymbol{h}]$ has the far-field asymptotic expansion

$$
\begin{aligned}
\boldsymbol{w}(\boldsymbol{x})= & |\boldsymbol{x}|^{-1} \sum_{p=1}^{3}(-1)^{p} \gamma_{p}\left(\widehat{\boldsymbol{\xi}}^{p}\right) E\left(\boldsymbol{\xi}^{p}\right)\left[\boldsymbol{b}\left(\boldsymbol{\xi}^{p}\right) \cdot \boldsymbol{I}^{p}(\widehat{\boldsymbol{x}})\right] e^{\mathrm{i} \varrho \widehat{\boldsymbol{x}} \cdot \boldsymbol{\xi}^{p}} \boldsymbol{b}\left(\boldsymbol{\xi}^{p}\right) \\
& +O\left(|\boldsymbol{x}|^{-2}\right)
\end{aligned}
$$

$$
\begin{aligned}
\boldsymbol{t}_{0}[\boldsymbol{w}](\boldsymbol{x})= & \mathrm{i}|\boldsymbol{x}|^{-1} \sum_{q=1}^{3}(-1)^{q} \gamma_{p}\left(\widehat{\boldsymbol{\xi}}^{p}\right) E\left(\boldsymbol{\xi}^{q}\right)\left[\boldsymbol{b}\left(\boldsymbol{\xi}^{p}\right) \cdot \boldsymbol{I}^{p}(\widehat{\boldsymbol{x}})\right] e^{\mathrm{i} \varrho \widehat{\boldsymbol{x}} \boldsymbol{\xi}^{q}} \\
& \widehat{\boldsymbol{A}}\left(\widehat{\boldsymbol{x}}, \boldsymbol{\xi}^{q}\right) \cdot \boldsymbol{b}\left(\boldsymbol{\xi}^{p}\right)+O\left(|\boldsymbol{x}|^{-2}\right)
\end{aligned}
$$

with $\boldsymbol{\xi}^{p}=\boldsymbol{\xi}^{p}(\widehat{\boldsymbol{x}}) \in S^{p}$ as defined after Assumption 1.1 and with the vectors $\boldsymbol{I}^{p}(\widehat{\boldsymbol{x}})$ defined by

$$
\boldsymbol{I}^{p}(\widehat{\boldsymbol{x}})=\int_{D_{1}} e^{-\mathrm{i} \boldsymbol{y} \cdot \boldsymbol{\xi}^{p}}[\boldsymbol{g}+\operatorname{div} \boldsymbol{h}](\boldsymbol{y}) d V_{y}-\int_{\Gamma} e^{-\mathrm{i} \boldsymbol{y} \cdot \boldsymbol{\xi}^{p}} \boldsymbol{h}(\boldsymbol{y}) \cdot \boldsymbol{n}(\boldsymbol{y}) d S_{y} .
$$

Proof. The lemma follows directly from the far-field asymptotic form $(2.4 \mathrm{a}),(2.4 \mathrm{~b})$ of the fundamental elastodynamic tensor and the equivalent representation $\boldsymbol{w}=\mathcal{V}_{\omega}[\boldsymbol{g}+\operatorname{div} \boldsymbol{h}]-\mathcal{S}_{\omega}[\boldsymbol{h} \cdot \boldsymbol{n}]$ of $\boldsymbol{w}$ stemming from (2.6).

Lemma 2.3. Let $S_{\varrho}$ denote the sphere $\left\{\boldsymbol{x} \in \mathbb{R}^{3},|\boldsymbol{x}|=\varrho\right\}$ of radius $\varrho$. 
(a) The integrals

$$
I_{R, k}:=R^{-1} \int_{R}^{2 R}\left\{\int_{S_{\varrho}} \boldsymbol{G}^{k}(\boldsymbol{x}) \cdot \overline{\boldsymbol{T}}^{k}(\boldsymbol{x}) d S_{x}\right\} d \varrho
$$

are such that $\Im\left(I_{R, k}\right)<0$ for $R$ large enough.

(b) For any displacement field $\boldsymbol{w}$ of the form $\boldsymbol{w}=\mathcal{V}_{\omega}[\boldsymbol{g}]+\mathcal{W}_{\omega}[\boldsymbol{h}]$, define $I_{R}(\boldsymbol{w})$ by

$$
I_{R}(\boldsymbol{w}):=R^{-1} \int_{R}^{2 R}\left\{\int_{S_{\varrho}} \boldsymbol{w}(\boldsymbol{x}) \cdot \boldsymbol{t}_{0}[\overline{\boldsymbol{w}}](\boldsymbol{x}) d S_{x}\right\} d \varrho .
$$

We have

$$
\begin{aligned}
I_{R}(\boldsymbol{w})= & -\mathrm{i} \sum_{p=1}^{3}(-1)^{p} \int_{\hat{S}} \gamma_{p}\left(\widehat{\boldsymbol{\xi}}^{p}\right)\left(2 \pi^{2}\left|\boldsymbol{\nabla} D\left(\boldsymbol{\xi}^{p}\right)\right| \kappa\left(\boldsymbol{\xi}^{p}\right)\right)^{-1} \\
& \times\left|\boldsymbol{I}^{p}(\widehat{\boldsymbol{x}}) \cdot \boldsymbol{b}^{p}\left(\widehat{\boldsymbol{\xi}}^{p}\right)\right|^{2} d S_{\hat{x}}+o(1),
\end{aligned}
$$

with $\boldsymbol{\xi}^{p}=\boldsymbol{\xi}^{p}(\widehat{\boldsymbol{x}}) \in S^{p}$ as defined after Assumption 1.1. In particular, $\Im\left(I_{R}\right)<0$ for $R$ large enough.

Proof. See subsection 6.1.

Remark 2.4. Lemma 2.3 (a) confirms that the elastodynamic state associated with each $G^{k}$ is radiating in the physical sense: the time average over one period of the energy flux across $S_{\varrho}$ averaged over $R \leq \varrho \leq 2 R$ is negative for large enough $R$ (see also Remark 6.1).

Moreover, upon replacing $\omega+\mathrm{i} \epsilon$ with $\omega-\mathrm{i} \epsilon$ in the integrand of $(2.2), \boldsymbol{G}$ represents incoming, rather than outgoing, waves (see [31] for details); this change, in particular, manifests itself through the substitution $\mathrm{i} \rightarrow-\mathrm{i}$ in $(2.4 \mathrm{a})$ and $(2.4 \mathrm{~b})$.

Then, part (b) shows that any $\boldsymbol{w}=\mathcal{V}_{\omega}[\boldsymbol{g}]+\mathcal{W}_{\omega}[\boldsymbol{h}]$ is radiating in the physical sense. The strict inequality in the last statement therein requires that the leading $O(1)$ contribution to $I_{R}(\boldsymbol{w})$ does not vanish. The uniqueness study of subsection 3.3 implies that any nonvanishing $\boldsymbol{w}$ in fact satisfies this condition.

2.3. Radiating transmission solutions and volume integral equation. We now establish a representation identity for transmission 
solutions in terms of volume potentials, from which the volume integral equation will follow. This step entails accounting for the behavior at infinity of transmission solutions and, in particular, defining what a radiating transmission solution is in the present context. We follow for that purpose the approach of [12] and [29, Chapter 7]. Accordingly, for $\boldsymbol{u}$ solving $\mathcal{B}_{0} \boldsymbol{u}=\mathbf{0}$ in $D_{0}$, let $\boldsymbol{\mathcal { M }}[\boldsymbol{u}](\boldsymbol{x})$ be defined for any given $\boldsymbol{x} \in D_{0}$ by

$$
\mathcal{M}[\boldsymbol{u}](\boldsymbol{x}):=\int_{S_{A}}\left(\boldsymbol{G}(\cdot-\boldsymbol{x}) \cdot \boldsymbol{t}_{0}[\boldsymbol{u}]-\boldsymbol{T}(\cdot-\boldsymbol{x}) \cdot \boldsymbol{u}\right) \mathrm{d} S
$$

(where $S_{A}$ is the sphere of radius $A$ centered at the origin) for any $A>|\boldsymbol{x}|$. This definition does not depend on the choice of (large enough) $A$ : for any $A^{\prime}>A$, the first Green's identity applied to the elastodynamic states $\boldsymbol{u}$ and $\boldsymbol{G}(\cdot-\boldsymbol{x})$, which verifies $\boldsymbol{B}_{0} \boldsymbol{u}=\mathbf{0}$ and $\mathcal{B}_{0} \boldsymbol{G}(\cdot-\boldsymbol{x})=\mathbf{0}$ in the region enclosed by the spheres $S_{A}$ and $S_{A^{\prime}}$, implies (using the exterior unit normal on both spheres $S_{A}$ and $S_{A^{\prime}}$ )

$$
\begin{aligned}
\int_{S_{A^{\prime}}}\left(\boldsymbol{G}(\cdot-\boldsymbol{x}) \cdot \boldsymbol{t}_{0}[\boldsymbol{u}]-\right. & \boldsymbol{T}(\cdot-\boldsymbol{x}) \cdot \boldsymbol{u}) \mathrm{d} S \\
= & \int_{S_{A}}\left(\boldsymbol{G}(\cdot-\boldsymbol{x}) \cdot \boldsymbol{t}_{0}[\boldsymbol{u}]-\boldsymbol{T}(\cdot-\boldsymbol{x}) \cdot \boldsymbol{u}\right) \mathrm{d} S .
\end{aligned}
$$

Lemma 2.5. Let the scattered field $\boldsymbol{v} \in \boldsymbol{H}_{\mathrm{loc}}^{1}\left(\mathbb{R}^{3}\right)$ satisfy the field equations (1.5) and transmission conditions (1.6) for some given incident field $\boldsymbol{u}_{I}$. Then, the value of the total field $\boldsymbol{u}=\boldsymbol{u}_{I}+\boldsymbol{v}$ at any point $\boldsymbol{x} \in D_{0} \cup D_{1}$ is given by the representation formula

$$
\boldsymbol{u}(\boldsymbol{x})=\mathcal{W}_{\omega}\left[\Delta \mathcal{C}: \varepsilon\left[\boldsymbol{u}_{1}\right]\right](\boldsymbol{x})+\omega^{2} \mathcal{V}_{\omega}\left[\Delta \rho \boldsymbol{u}_{1}\right](\boldsymbol{x})+\boldsymbol{u}_{I}(\boldsymbol{x})+\mathcal{M}\left[\boldsymbol{v}_{0}\right](\boldsymbol{x})
$$

Proof. Consider a bounded domain $X \subset \mathbb{R}^{3}$, and let the partial differential operator $\mathcal{B}$ be defined (in the distributional sense) by $\mathcal{B} \boldsymbol{w}=-\operatorname{div}(\mathcal{C}: \boldsymbol{\varepsilon}[\boldsymbol{w}])-\rho \omega^{2} \boldsymbol{w}$ for some elasticity tensor $\mathcal{C}$ and mass density $\rho$. Let $\boldsymbol{w} \in \boldsymbol{H}^{1}(X)$ be such that $\boldsymbol{B} \boldsymbol{w}=\mathbf{0}$ in $X$, and define the distribution $\widetilde{\boldsymbol{w}}$ as the extension of $\boldsymbol{w}$ by zero in $\mathbb{R}^{3} \backslash \bar{X}$. For any test function $\varphi \in C_{0}^{\infty}\left(\mathbb{R}^{3} ; \mathbb{C}^{3}\right)$, one has

$$
\begin{aligned}
\left(\mathcal{B}_{0} \widetilde{\boldsymbol{w}}, \boldsymbol{\varphi}\right) & =\left(\widetilde{\boldsymbol{w}},\left(\mathcal{B}_{0}-\mathcal{B}\right) \boldsymbol{\varphi}\right)+(\widetilde{\boldsymbol{w}}, \mathcal{B} \boldsymbol{\varphi}) \\
& =\left(\boldsymbol{w},\left(\mathcal{B}_{0}-\mathcal{B}\right) \boldsymbol{\varphi}\right)_{X}+(\boldsymbol{w}, \mathcal{B} \boldsymbol{\varphi})_{X}
\end{aligned}
$$


(with $(\cdot, \cdot)$ and $(\cdot, \cdot)_{X}$ respectively denoting the distributional duality product and the $\boldsymbol{L}^{2}(X)$ scalar product), since $\boldsymbol{B}_{0}$ involves only evenorder derivatives. Next, using the definition of operators $\mathcal{B}$ and $\boldsymbol{B}_{0}$ and the first and second Green identities [29, Theorem 4.4] for the domain $X$, one has

$$
\begin{aligned}
\left(\boldsymbol{w},\left(\mathcal{B}_{0}-\mathcal{B}\right) \boldsymbol{\varphi}\right)_{X} & =\left(\boldsymbol{w}, \boldsymbol{t}[\boldsymbol{\varphi}]-\boldsymbol{t}_{0}[\boldsymbol{\varphi}]\right)_{\partial X}-\langle\boldsymbol{w}, \boldsymbol{\varphi}\rangle_{X}^{\mathcal{C}-\mathcal{C}_{0}}+\omega^{2}(\boldsymbol{w}, \boldsymbol{\varphi})_{X}^{\rho-\rho_{0}} \\
(\boldsymbol{w}, \mathcal{B} \boldsymbol{\varphi})_{X} & =-(\boldsymbol{w}, \boldsymbol{t}[\boldsymbol{\varphi}])_{\partial X}+(\boldsymbol{t}[\boldsymbol{w}], \boldsymbol{\varphi})_{\partial X}
\end{aligned}
$$

$(\boldsymbol{\varphi} \rightarrow \boldsymbol{t}[\boldsymbol{\varphi}]$ denoting the surface traction operator relative to elastic properties $\mathcal{C}$, and, with the second equality using the formal selfadjointness of $\mathcal{B}$ and $\mathcal{B} \boldsymbol{w}=\mathbf{0}$ in $X$ ), therefore

$$
\left(\boldsymbol{B}_{0} \widetilde{\boldsymbol{w}}, \boldsymbol{\varphi}\right)=-\left(\boldsymbol{w}, \boldsymbol{t}_{0}[\boldsymbol{\varphi}]\right)_{\partial X}+(\boldsymbol{t}[\boldsymbol{w}], \boldsymbol{\varphi})_{\partial X}-\langle\boldsymbol{w}, \boldsymbol{\varphi}\rangle_{X}^{\mathcal{C}-\mathcal{C}_{0}}+\omega^{2}(\boldsymbol{w}, \boldsymbol{\varphi})_{X}^{\rho-\rho_{0}}
$$

Equality (2.10) holds for arbitrarily chosen bounded domain $X \subset \mathbb{R}^{3}$ and physically acceptable material properties $\mathcal{C}, \rho$. It is now applied (i) for $X=D_{1}$ with $\boldsymbol{w}=\boldsymbol{u}_{1}$ and $\mathcal{B}=\mathcal{B}_{1}$, and (ii) for $X=D_{0} \cap B_{R}$ with $\boldsymbol{w}=\boldsymbol{u}_{0}$ and $\mathcal{B}=\mathcal{B}_{0}$ (where $B_{R}$ is the ball of radius $R$ centered at the origin, bounded by the sphere $S_{R}$ ), yielding the identities

$$
\begin{aligned}
& \left(\mathcal{B}_{0} \widetilde{\boldsymbol{u}}_{1}, \boldsymbol{\varphi}\right)=-\left(\boldsymbol{u}_{1}, \boldsymbol{t}_{0}[\boldsymbol{\varphi}]\right)_{\Gamma}+\left(\boldsymbol{t}_{1}\left[\boldsymbol{u}_{1}\right], \boldsymbol{\varphi}\right)_{\Gamma}-\left\langle\boldsymbol{u}_{1}, \boldsymbol{\varphi}\right\rangle_{D_{1}}^{\Delta \mathcal{C}}+\omega^{2}\left(\boldsymbol{u}_{1}, \boldsymbol{\varphi}\right)_{D_{1}}^{\Delta \rho} \\
& \left(\boldsymbol{B}_{0} \widetilde{\boldsymbol{u}}_{0}, \boldsymbol{\varphi}\right)=\left(\boldsymbol{u}_{0}, \boldsymbol{t}_{0}[\boldsymbol{\varphi}]\right)_{\Gamma}-\left(\boldsymbol{t}_{0}\left[\boldsymbol{u}_{0}\right], \boldsymbol{\varphi}\right)_{\Gamma}-\left(\boldsymbol{u}_{0}, \boldsymbol{t}_{0}[\boldsymbol{\varphi}]\right)_{S_{R}}+\left(\boldsymbol{t}_{0}\left[\boldsymbol{u}_{0}\right], \boldsymbol{\varphi}\right)_{S_{R}}
\end{aligned}
$$

(the sign inversion for integrals over $\Gamma$ in the second equality being caused by the unit normal to $\Gamma$ conventionally pointing inwards of $D_{0}$ ). Summing those equalities and invoking the transmission conditions (1.6) gives

$$
\begin{aligned}
\left(\mathcal{B}_{0}\left(\widetilde{\boldsymbol{u}}_{0}+\widetilde{\boldsymbol{u}}_{1}\right), \boldsymbol{\varphi}\right)= & -\left\langle\boldsymbol{u}_{1}, \boldsymbol{\varphi}\right\rangle_{D_{1}}^{\Delta \mathcal{C}}+\omega^{2}\left(\boldsymbol{u}_{1}, \boldsymbol{\varphi}\right)_{D_{1}}^{\Delta \rho} \\
& -\left(\boldsymbol{u}_{0}, \boldsymbol{t}_{0}[\boldsymbol{\varphi}]\right)_{S_{R}}+\left(\boldsymbol{t}_{0}\left[\boldsymbol{u}_{0}\right], \boldsymbol{\varphi}\right)_{S_{R}} .
\end{aligned}
$$

Equality (2.11) holds for any test function $\varphi \in C_{0}^{\infty}\left(\mathbb{R}^{3} ; \mathbb{C}^{3}\right)$, so it is an equality between two distributions whose supports are compact. One can then take the distributional convolution of both members by the fundamental tensor $\boldsymbol{G}$, which satisfies $\boldsymbol{B}_{0} \boldsymbol{G}=\delta \boldsymbol{I}$, to obtain

$$
\begin{aligned}
\left(\widetilde{\boldsymbol{u}}_{0}+\widetilde{\boldsymbol{u}}_{1}, \boldsymbol{\varphi}\right)= & -\left\langle\boldsymbol{u}_{1}, \boldsymbol{G} \star \boldsymbol{\varphi}\right\rangle_{D_{1}}^{\Delta \mathcal{C}}+\omega^{2}\left(\boldsymbol{u}_{1}, \boldsymbol{G} \star \boldsymbol{\varphi}\right)_{D_{1}}^{\Delta \rho} \\
& -\left(\boldsymbol{u}_{0}, \boldsymbol{t}_{0}[\boldsymbol{G} \star \boldsymbol{\varphi}]\right)_{S_{R}}+\left(\boldsymbol{t}_{0}\left[\boldsymbol{u}_{0}\right], \boldsymbol{G} \star \boldsymbol{\varphi}\right)_{S_{R}},
\end{aligned}
$$


where the left-hand side results from $\boldsymbol{G} \star \mathcal{B}_{0}\left(\widetilde{\boldsymbol{u}}_{0}+\widetilde{\boldsymbol{u}}_{1}\right)=\boldsymbol{\mathcal { B }}_{0} \boldsymbol{G} \star\left(\widetilde{\boldsymbol{u}}_{0}+\widetilde{\boldsymbol{u}}_{1}\right)=$ $\widetilde{\boldsymbol{u}}_{0}+\widetilde{\boldsymbol{u}}_{1}$ while the right-hand side stems from $(\boldsymbol{G} \star \boldsymbol{w}, \boldsymbol{\varphi})=(\boldsymbol{w}, \boldsymbol{G} \star \boldsymbol{\varphi})$ for any compactly supported distribution $\boldsymbol{w} \in \mathcal{D}^{\prime}\left(\mathbb{R}^{3} ; \mathbb{C}^{3}\right)$; note that here the convolution $\star$ entails an inner product, e.g., $\boldsymbol{G} \star \boldsymbol{\varphi}=\int_{\mathbb{R}^{3}} \boldsymbol{G}(\cdot-$ $\boldsymbol{x}) \cdot \boldsymbol{\varphi}(\boldsymbol{x}) \mathrm{d} V(\boldsymbol{x})$.

The remaining task is to evaluate each term on the right-hand side of (2.12). We have

$$
\begin{aligned}
\left(\boldsymbol{u}_{1}, \boldsymbol{G} \star \boldsymbol{\varphi}\right)_{D_{1}}^{\Delta \rho} & =\int_{D_{1}} \Delta \rho(\boldsymbol{y}) \boldsymbol{u}_{1}(\boldsymbol{y}) \cdot\left\{\int_{\mathbb{R}^{3}} \boldsymbol{G}(\boldsymbol{y}-\boldsymbol{x}) \cdot \boldsymbol{\varphi}(\boldsymbol{x}) \mathrm{d} V(\boldsymbol{x})\right\} \mathrm{d} V(\boldsymbol{y}) \\
& =\int_{\mathbb{R}^{3}}\left\{\int_{D_{1}} \Delta \rho(\boldsymbol{y}) \boldsymbol{G}(\boldsymbol{x}-\boldsymbol{y}) \cdot \boldsymbol{u}_{1}(\boldsymbol{y}) \mathrm{d} V(\boldsymbol{y})\right\} \cdot \boldsymbol{\varphi}(\boldsymbol{x}) \mathrm{d} V(\boldsymbol{x}) \\
& =\left(\mathcal{V}_{\omega}\left[\Delta \rho \boldsymbol{u}_{1}\right], \boldsymbol{\varphi}\right)
\end{aligned}
$$

(the second equality exploiting the symmetry properties (2.3) (a) of $\boldsymbol{G}$ ) and, similarly,

$$
\begin{aligned}
\left\langle\boldsymbol{u}_{1}, \boldsymbol{G} \star \boldsymbol{\varphi}\right\rangle_{D_{1}}^{\Delta \mathcal{C}}= & \int_{D_{1}} \boldsymbol{\nabla} \boldsymbol{u}_{1}(\boldsymbol{y}): \Delta \mathcal{C}(\boldsymbol{y}): \\
& \boldsymbol{\nabla}\left\{\int_{\mathbb{R}^{3}} \boldsymbol{G}(\boldsymbol{y}-\boldsymbol{x}) \cdot \boldsymbol{\varphi}(\boldsymbol{x}) \mathrm{d} V(\boldsymbol{x})\right\} \mathrm{d} V(\boldsymbol{y}) \\
= & -\int_{\mathbb{R}^{3}}\left\{\int_{D_{1}} \boldsymbol{\nabla} \boldsymbol{G}(\boldsymbol{x}-\boldsymbol{y}): \Delta \mathcal{C}(\boldsymbol{y}): \boldsymbol{\nabla} \boldsymbol{u}_{1}(\boldsymbol{y}) \mathrm{d} V(\boldsymbol{y})\right\} \\
& \cdot \boldsymbol{\varphi}(\boldsymbol{x}) \mathrm{d} V(\boldsymbol{x}) \\
= & -\left(\mathcal{W}_{\omega}\left[\Delta \mathcal{C}: \varepsilon\left[\boldsymbol{u}_{1}\right]\right], \boldsymbol{\varphi}\right)
\end{aligned}
$$

(the second equality again exploiting properties (2.3) (a)). Finally, the last two terms on the right-hand side of $(2.12)$ are evaluated under the additional assumption that $\varphi \in C_{0}^{\infty}\left(B_{R} ; \mathbb{C}^{3}\right)$, so that

$$
\begin{aligned}
- & \left(\boldsymbol{u}_{0}, \boldsymbol{t}_{0}[\boldsymbol{G} \star \boldsymbol{\varphi}]\right)_{S_{R}}+\left(\boldsymbol{t}_{0}\left[\boldsymbol{u}_{0}\right], \boldsymbol{G} \star \boldsymbol{\varphi}\right)_{S_{R}} \\
= & \int_{S_{R}}\left(\boldsymbol{t}_{0}\left[\boldsymbol{u}_{0}\right](\boldsymbol{y}) \cdot\left\{\int_{\mathbb{R}^{3}} \boldsymbol{G}(\boldsymbol{y}-\boldsymbol{x}) \cdot \boldsymbol{\varphi}(\boldsymbol{x}) \mathrm{d} V(\boldsymbol{x})\right\}-\boldsymbol{u}_{0}(\boldsymbol{y})\right. \\
& \left.\cdot \boldsymbol{t}_{0}\left[\int_{\mathbb{R}^{3}} \boldsymbol{G}(\boldsymbol{y}-\boldsymbol{x}) \cdot \boldsymbol{\varphi}(\boldsymbol{x}) \mathrm{d} V(\boldsymbol{x})\right]\right) \mathrm{d} S(\boldsymbol{y}) \\
= & \int_{\mathbb{R}^{3}}\left\{\int_{S_{R}}\left(\boldsymbol{t}_{0}\left[\boldsymbol{u}_{0}\right](\boldsymbol{y}) \cdot \boldsymbol{G}(\boldsymbol{y}-\boldsymbol{x})-\boldsymbol{u}_{0}(\boldsymbol{y}) \cdot \boldsymbol{T}(\boldsymbol{y}-\boldsymbol{x})\right) \mathrm{d} S(\boldsymbol{y})\right\}
\end{aligned}
$$




$$
\begin{aligned}
& \cdot \boldsymbol{\varphi}(\boldsymbol{x}) \mathrm{d} V(\boldsymbol{x}) \\
= & \left(\boldsymbol{M}\left[\boldsymbol{u}_{0}\right], \boldsymbol{\varphi}\right), \quad \text { for all } \boldsymbol{\varphi} \in C_{0}^{\infty}\left(B_{R} ; \mathbb{C}^{3}\right) .
\end{aligned}
$$

Inserting the last three identities into (2.12), sending $R$ to infinity and noting that the resulting distributions are locally summable functions, we obtain

$$
\boldsymbol{u}=\mathcal{W}_{\omega}\left[\Delta \mathcal{C}: \varepsilon\left[\boldsymbol{u}_{1}\right]\right]+\omega^{2} \mathcal{V}_{\omega}\left[\Delta \rho \boldsymbol{u}_{1}\right]+\mathcal{M}\left[\boldsymbol{u}_{0}\right] \quad \text { in } D_{0} \cup D_{1}
$$

Finally, since $\boldsymbol{u}_{\mathrm{I}}$ solves $\boldsymbol{B}_{0} \boldsymbol{u}_{\mathrm{I}}=\mathbf{0}$ in $\mathbb{R}^{3}$ (in which case $\Delta \mathcal{C}=\mathbf{0}$ and $\Delta \rho=0$ ), applying equality (2.10) to $X=B_{R}$ and $\boldsymbol{w}=\left.\boldsymbol{u}_{\mathrm{I}}\right|_{B_{R}}$ and subsequent convolution by the fundamental tensor $\boldsymbol{G}$ provides

$$
\mathcal{M}\left[\boldsymbol{u}_{\mathrm{I}}\right]=\boldsymbol{u}_{\mathrm{I}}, \quad \text { i.e., } \mathcal{M}\left[\boldsymbol{u}_{0}\right]=\boldsymbol{u}_{\mathrm{I}}+\mathcal{M}\left[\boldsymbol{v}_{0}\right] .
$$

This completes the proof of the lemma.

When defined in terms of the radiating Green's tensor, $\mathcal{M}[\boldsymbol{v}]$ is the "contribution from infinity" to a displacement $\boldsymbol{v}$, i.e., its "nonradiating" part. In usual contexts such as linear acoustics, electromagnetism or isotropic elasticity, radiating solutions are defined by enforcing relevant Sommerfeld-type radiation conditions at infinity, which is in fact equivalent to setting $\mathcal{M}[\boldsymbol{v}]=\mathbf{0}$ (see, e.g., [29, Chapter 9] for acoustics, and Section 4). Since radiation conditions are rather involved for general anisotropic media $[\mathbf{3 1}, \mathbf{3 6}]$ or configurations such as semi-infinite media, we choose here to adopt the latter definition:

Definition 2.6. A solution $\boldsymbol{v}$ of $\mathcal{B}_{0} \boldsymbol{v}=\mathbf{0}$ in $D_{0}$ is radiating if $\mathcal{M}[v]=\mathbf{0}$.

Remark 2.7. The viewpoint of Definition 2.6 is, for example, adopted in [27], where elastodynamic problems for layered semi-infinite media are considered.

Lemma 2.5 implies that radiating solutions (in the sense of Definition 2.6) of the transmission problems (1.5) and (1.6) satisfy a volume integro-differential equation of the Lippmann-Schwinger type: 
Proposition 2.8 (Lippmann-Schwinger integral equation). Define the integral operator $\mathcal{A}_{\omega}: \boldsymbol{H}^{1}\left(D_{1}\right) \rightarrow \boldsymbol{H}^{1}\left(D_{1}\right)$ by

$$
\mathcal{A}_{\omega}[\boldsymbol{w}](\boldsymbol{x})=\mathcal{W}_{\omega}[\Delta \mathcal{C}: \varepsilon[\boldsymbol{w}]](\boldsymbol{x})+\omega^{2} \mathcal{V}_{\omega}[\Delta \rho \boldsymbol{w}](\boldsymbol{x}), \quad \boldsymbol{x} \in D_{1}
$$

(a) For any solution $\boldsymbol{v}$ of the transmission problems (1.5) and (1.6) that are radiating (in the sense of Definition 2.6), the total field $\boldsymbol{u}_{1}=\boldsymbol{u}_{I}+\boldsymbol{v}_{1}$ satisfies the integral equation

$$
\left(\mathcal{I}-\mathcal{A}_{\omega}\right) \boldsymbol{u}_{1}(\boldsymbol{x})=\boldsymbol{u}_{I}(\boldsymbol{x}) \quad\left(\boldsymbol{x} \in D_{1}\right),
$$

with $\mathcal{I}$ denoting the identity operator.

(b) Then, $\boldsymbol{u}_{0}=\boldsymbol{u}_{I}+\boldsymbol{v}_{0}$ is given explicitly in terms of $\boldsymbol{u}_{1}$ by the integral representation formula

$$
\begin{gathered}
\boldsymbol{u}_{0}(\boldsymbol{x})=\boldsymbol{u}_{I}(\boldsymbol{x})+\mathcal{W}_{\omega}\left[\Delta \mathcal{C}: \varepsilon\left[\boldsymbol{u}_{1}\right]\right](\boldsymbol{x})+\omega^{2} \mathcal{V}_{\omega}\left[\Delta \rho \boldsymbol{u}_{1}\right](\boldsymbol{x}) \\
\left(\boldsymbol{x} \in D_{0}\right) .
\end{gathered}
$$

The main concern of this work is then to establish that the volume integral equation (2.14) is uniquely solvable and is equivalent to seeking radiating solutions to the initial transmission problems (1.5) and (1.6).

3. Solvability of the volume integral equation. To investigate the solvability of integral equation (2.14), we begin by establishing relevant properties of the volume potentials and the Fredholm character of the integral operator, following the steps used in $[\mathbf{2 1 ,} \mathbf{2 2}, \mathbf{2 3}, \mathbf{2 4}]$ for transmission problems involving the Helmholtz or Maxwell equations. The solvability result (Theorem 3.10) will then follow from showing that the homogeneous transmission problem has at most one radiating solution.

\subsection{Volume potentials as radiating solutions.}

Lemma 3.1. The volume potentials $\mathcal{V}_{\omega}$ and $\mathcal{W}_{\omega}$ defined by equations (2.5a), (2.5b) are radiating (in the sense of Definition 2.6).

Proof. We first consider the case of $\mathcal{V}_{\omega}$, and set $\boldsymbol{w}:=\mathcal{V}_{\omega}[\boldsymbol{g}]$. Using the definition (2.5a) of $\mathcal{V}_{\omega}[\boldsymbol{g}]$ in equation (2.9) and effecting some 
manipulation, we obtain

$$
\begin{aligned}
\mathcal{M}[\boldsymbol{w}](\boldsymbol{x})= & \int_{D_{1}}\left\{\int_{S_{A}}\left(\boldsymbol{G}(\boldsymbol{z}-\boldsymbol{x}) \cdot \boldsymbol{T}(\boldsymbol{z}-\boldsymbol{y})-\boldsymbol{T}^{\mathrm{T}}(\boldsymbol{z}-\boldsymbol{x}) \cdot \boldsymbol{G}(\boldsymbol{z}-\boldsymbol{y})\right) \mathrm{d} S(\boldsymbol{z})\right\} \\
& \cdot \boldsymbol{g}(\boldsymbol{y}) \mathrm{d} V(\boldsymbol{y}) .
\end{aligned}
$$

Then, observing that $\boldsymbol{G}(\boldsymbol{z}-\boldsymbol{x}) \cdot \boldsymbol{T}(\boldsymbol{z}-\boldsymbol{y})-\boldsymbol{T}^{\mathrm{T}}(\boldsymbol{z}-\boldsymbol{x}) \cdot \boldsymbol{G}(\boldsymbol{z}-\boldsymbol{y})=O\left(A^{-3}\right)$ as $A=|\boldsymbol{z}| \rightarrow \infty$ (by virtue of the far-field asymptotic formulas (2.4a) and (2.4b), and noting that the matrix $\boldsymbol{N}\left(\boldsymbol{\xi}^{q}\right)$ is symmetric), we obtain $\mathcal{M}[\boldsymbol{w}](\boldsymbol{x})=\mathbf{0}$ since (i) $\mathcal{M}$ as defined by equation (2.9) is independent on $A$ for large enough $A$ and (ii) the above integral over $S_{A}$ is $O\left(A^{-1}\right)$, $D_{1}$ is bounded and $\boldsymbol{g} \in L^{2}\left(D_{1}, \mathbb{C}^{3}\right)$.

Essentially the same proof applies to $\mathcal{S}_{\omega}$, and hence to $\mathcal{W}_{\omega}$ by virtue of equation (2.6).

Lemma 3.2. Consider the volume potentials $\mathcal{V}_{\omega}$ and $\mathcal{W}_{\omega}$, as defined by equations (2.5a) and (2.5b), for respective densities $\boldsymbol{g} \in C^{0, \alpha}\left(D_{1} ; \mathbb{C}^{3}\right)$ and $\boldsymbol{h} \in C^{1, \alpha}\left(D_{1} ; \mathbb{C}^{3 \times 3}\right)$.

(i) The displacement $\boldsymbol{w}=\mathcal{V}_{\omega}[\boldsymbol{g}]+\mathcal{W}_{\omega}[\boldsymbol{h}]$ is a radiating solution in $\mathbb{R}^{3}$ of

$$
\begin{aligned}
& \text { (a) } \mathcal{B}_{0} \boldsymbol{w}=\left\{\begin{array}{r}
\boldsymbol{g}+\operatorname{div} \boldsymbol{h} \text { in } D_{1} \\
\mathbf{0} \quad \text { in } D_{0}
\end{array},\right. \\
& \text { (b) } \boldsymbol{w}_{0}=\boldsymbol{w}_{1}, \quad \boldsymbol{t}_{0}\left[\boldsymbol{w}_{0}\right]=\boldsymbol{t}_{0}\left[\boldsymbol{w}_{1}\right]+\boldsymbol{h} \cdot \boldsymbol{n} \quad \text { on } \Gamma .
\end{aligned}
$$

(ii) The above problem has the variational form

$$
\langle\boldsymbol{v}, \boldsymbol{\psi}\rangle_{\mathbb{R}^{3}}^{\mathcal{C}^{0}}-\omega^{2}(\boldsymbol{v}, \boldsymbol{\psi})_{\mathbb{R}^{3}}^{\rho^{0}}=-(\boldsymbol{h}, \boldsymbol{\nabla} \boldsymbol{\psi})_{D_{1}}+(\boldsymbol{g}, \boldsymbol{\psi})_{D_{1}}
$$

for all $\boldsymbol{\psi} \in \boldsymbol{H}_{\mathrm{comp}}^{1}\left(\mathbb{R}^{3}\right)$.

Proof. We know from Lemma 3.1 that $\boldsymbol{w}$ is radiating. To finish proving Lemma 3.2 (i), we first use the alternative expression (2.6) of $\mathcal{W}_{\omega}[\boldsymbol{h}]$. Recalling that $\boldsymbol{B}_{0} \mathcal{S}_{\omega}[\boldsymbol{f}]=\mathbf{0}$ in $D_{0} \cup D_{1}$, treating the volume potential as a convolution in the distributional sense, and using $\mathcal{B}_{0} \boldsymbol{G}=\delta \boldsymbol{I}$, one readily obtains

$$
\begin{aligned}
\mathcal{B}_{0} \boldsymbol{w} & =\mathcal{B}_{0}\left(\mathcal{V}_{\omega}[\boldsymbol{g}+\operatorname{div} \boldsymbol{h}]-\mathcal{S}_{\omega}[\boldsymbol{h} \cdot \boldsymbol{n}]\right) \\
& =\left[\mathcal{B}_{0} \boldsymbol{G}\right] \star\left[1_{D_{1}}(\boldsymbol{g}+\operatorname{div} \boldsymbol{h})\right]
\end{aligned}
$$




$$
=1_{D_{1}}(\boldsymbol{g}+\operatorname{div} \boldsymbol{h}) \quad \text { in } D_{0} \cup D_{1},
$$

which proves (a). For (b), single-layer and volume potentials with $C^{0, \alpha}$ densities are known to define $C^{0}\left(\mathbb{R}^{3}\right)$ and $C^{1}\left(\mathbb{R}^{3}\right)$ functions, respectively, see e.g., [15, Chapter 4] or [25, Chapter 4] for isotropic elastodynamics (the proof of [15], while expounded about the Laplace equation, relies only on the fact that $\boldsymbol{G} \in C^{\infty}\left(\mathbb{R}^{3} \backslash\{\mathbf{0}\} ; \mathbb{R}^{3 \times 3}\right)$ and has a $O\left(|\boldsymbol{x}|^{-1}\right)$ singularity at the origin, which therefore applies to the present case as well). Consequently,

$$
\boldsymbol{w}=\mathcal{V}_{\omega}[\boldsymbol{g}+\operatorname{div} \boldsymbol{h}]-\mathcal{S}_{\omega}[\boldsymbol{h} \cdot \boldsymbol{n}]
$$

is, in particular, continuous across $\Gamma$. Moreover, the derivatives of $\mathcal{V}_{\omega}[\boldsymbol{g}+\operatorname{div} \boldsymbol{h}]$ are also continuous across $\Gamma$ (in the sense of traces), and therefore so is its traction vector. Finally, the known jump properties of the conormal derivative of a single-layer potential [29, e.g., Theorem 6.11], which are valid for any strongly elliptic partial differential operator (the conormal derivative considered here being the traction operator (1.1)), yield

$$
\boldsymbol{t}_{0}\left[\mathcal{S}_{\omega}[\boldsymbol{h} \cdot \boldsymbol{n}]\right]\left(\boldsymbol{x}_{+}\right)=\boldsymbol{t}_{0}\left[\mathcal{S}_{\omega}[\boldsymbol{h} \cdot \boldsymbol{n}]\right]\left(\boldsymbol{x}_{-}\right)+\boldsymbol{h}(\boldsymbol{x}) \cdot \boldsymbol{n}(\boldsymbol{x}), \quad \boldsymbol{x} \in \Gamma .
$$

This completes the proof of transmission conditions (ii) verified by the displacement $\boldsymbol{w}$.

Part (ii) follows from the weak form $\left(\boldsymbol{B}_{0} \boldsymbol{w}, \boldsymbol{\psi}\right)_{D_{0}}+\left(\boldsymbol{B}_{0} \boldsymbol{w}, \boldsymbol{\psi}\right)_{D_{1}}=$ $(\boldsymbol{g}+\operatorname{div} \boldsymbol{h}, \boldsymbol{\psi})_{D_{1}}$ of (a), by applying the first Green identity to each term in the left-hand side, using the transmission conditions (b) and noting that $(\operatorname{div} \boldsymbol{h}, \boldsymbol{\psi})_{D_{1}}=(\boldsymbol{h} \cdot \boldsymbol{n}, \boldsymbol{\psi})_{\Gamma}-(\boldsymbol{h}, \boldsymbol{\nabla} \boldsymbol{\psi})_{D_{1}}$, by virtue of the first Green identity.

3.2. Fredholm character of the volume integral operator. Let the inner product $\left\langle\left\langle\boldsymbol{w}^{\prime}, \boldsymbol{w}^{\prime \prime}\right\rangle\right\rangle$ be defined for vector fields $\boldsymbol{w}^{\prime}, \boldsymbol{w}^{\prime \prime} \in$ $\boldsymbol{H}^{1}\left(D_{1}\right)$ by

$$
\left\langle\left\langle\boldsymbol{w}^{\prime}, \boldsymbol{w}^{\prime \prime}\right\rangle\right\rangle=\left\langle\boldsymbol{w}^{\prime}, \overline{\boldsymbol{w}}^{\prime \prime}\right\rangle_{D_{1}}^{\mathcal{C}^{0}+\mathcal{C}^{1}}+\left(\boldsymbol{w}^{\prime}, \overline{\boldsymbol{w}}^{\prime \prime}\right)_{D_{1}}^{\rho^{0}+\rho^{1}} .
$$

By virtue of Korn's inequality, $\left.\|\boldsymbol{w}\|_{\mathcal{H}\left(D_{1}\right)}:=\langle\boldsymbol{w}, \boldsymbol{w}\rangle\right\rangle^{1 / 2}$ then defines a norm. Let $\mathcal{H}\left(D_{1}\right)$ denote the completion of $\boldsymbol{H}^{1}\left(D_{1}\right)$ with respect to $\|\cdot\|_{\mathcal{H}\left(D_{1}\right)} \cdot$ 
Lemma 3.3. The operator $\mathcal{I}-\mathcal{A}_{\mathrm{i}}$ is coercive in $\mathcal{H}\left(D_{1}\right)$ :

$$
\Re\left\langle\left\langle\left(\mathcal{I}-\mathcal{A}_{\mathrm{i}}\right) \boldsymbol{v}, \overline{\boldsymbol{v}}\right\rangle \geq\langle\langle\boldsymbol{v}, \overline{\boldsymbol{v}}\rangle\right.
$$

for any $\boldsymbol{v} \in \mathcal{H}\left(D_{1}\right)$.

Proof. For $\boldsymbol{v} \in \mathcal{H}\left(D_{1}\right)$, define $\boldsymbol{w}$ by $\boldsymbol{w}=\mathcal{A}_{\mathrm{i}}[\boldsymbol{v}]$, which is of the form $\boldsymbol{w}=\mathcal{V}_{\omega}[\boldsymbol{g}]+\mathcal{W}_{\omega}[\boldsymbol{h}]$ considered in Lemma 3.2 with $\boldsymbol{g}=-\Delta \rho \boldsymbol{v}$, $\boldsymbol{h}=\Delta \mathcal{C}: \varepsilon[\boldsymbol{v}]$ and $\omega=\mathrm{i}$. Moreover, $\boldsymbol{w}$ has a fast decay at infinity such that, in particular, $\boldsymbol{w} \in \boldsymbol{H}^{1}\left(\mathbb{R}^{3}\right)$ (see subsection 6.2 for a proof). The variational equation (3.1) with $\omega=\mathrm{i}$ therefore takes the form

$$
\langle\boldsymbol{w}, \boldsymbol{\psi}\rangle_{\mathbb{R}^{3}}^{\mathcal{C}^{0}}+(\boldsymbol{w}, \boldsymbol{\psi})_{\mathbb{R}^{3}}^{\rho^{0}}=-(\boldsymbol{v}, \boldsymbol{\psi})_{D_{1}}^{\Delta \mathcal{C}}-(\boldsymbol{v}, \boldsymbol{\psi})_{D_{1}}^{\Delta \rho}
$$

for any $\boldsymbol{\psi} \in \boldsymbol{H}^{1}\left(\mathbb{R}^{3}\right)$. Then, starting from the definition of the inner product $\langle$, $\rangle$ and exploiting the above variational equation with $\psi=\overline{\boldsymbol{w}}$, we have

$$
\begin{aligned}
\Re\left\langle\left(\mathcal{I}-\mathcal{A}_{\mathrm{i}}\right) \boldsymbol{v}, \overline{\boldsymbol{v}}\right\rangle= & \Re\langle\langle\boldsymbol{v}-\boldsymbol{w}, \overline{\boldsymbol{v}}\rangle \\
= & \langle\boldsymbol{v}, \overline{\boldsymbol{v}}\rangle_{D_{1}}^{\mathcal{C}^{0}+\mathcal{C}^{1}}+(\boldsymbol{v}, \overline{\boldsymbol{v}})_{D_{1}}^{\rho^{0}+\rho^{1}} \\
& -\Re\langle\boldsymbol{w}, \overline{\boldsymbol{v}}\rangle_{D_{1}}^{\mathcal{C}^{0}+\mathcal{C}^{1}}-\Re(\boldsymbol{w}, \overline{\boldsymbol{v}})_{D_{1}}^{\rho^{0}+\rho^{1}} \\
= & \langle\boldsymbol{v}, \overline{\boldsymbol{v}}\rangle_{D_{1}}^{\mathcal{C}^{0}+\mathcal{C}^{1}}+(\boldsymbol{v}, \overline{\boldsymbol{v}})_{D_{1}}^{\rho^{0}+\rho^{1}}-2 \Re\langle\boldsymbol{w}, \overline{\boldsymbol{v}}\rangle_{D_{1}}^{\mathcal{C}^{0}}-2 \Re(\boldsymbol{w}, \overline{\boldsymbol{v}})_{D_{1}}^{\rho^{0}} \\
& +\langle\boldsymbol{w}, \overline{\boldsymbol{w}}\rangle_{\mathbb{R}^{3}}^{\mathcal{C}^{0}}+(\boldsymbol{w}, \overline{\boldsymbol{w}})_{\mathbb{R}^{3}}^{\rho^{0}} \\
= & \langle\boldsymbol{v}, \overline{\boldsymbol{v}}\rangle_{D_{1}}^{\mathcal{C}^{1}}+(\boldsymbol{v}, \overline{\boldsymbol{v}})_{D_{1}}^{\rho^{1}}+\langle\boldsymbol{w}-\boldsymbol{v}, \overline{\boldsymbol{w}}-\overline{\boldsymbol{v}}\rangle_{D_{1}}^{\mathcal{C}^{0}} \\
& +(\boldsymbol{w}-\boldsymbol{v}, \overline{\boldsymbol{w}}-\overline{\boldsymbol{v}})_{D_{1}}^{\rho^{0}} \\
\geq & \langle\boldsymbol{v}, \overline{\boldsymbol{v}}\rangle_{D_{1}}^{\mathcal{C}^{1}}+(\boldsymbol{v}, \overline{\boldsymbol{v}})_{D_{1}}^{\rho^{1}} .
\end{aligned}
$$

The desired coercivity finally follows from the fact that $\left[\langle\boldsymbol{v}, \overline{\boldsymbol{v}}\rangle_{D_{1}}^{\mathcal{C}^{1}}+\right.$ $\left.(\boldsymbol{v}, \overline{\boldsymbol{v}})_{D_{1}}^{\rho^{1}}\right]^{1 / 2}$ and $\|\boldsymbol{v}\|_{\mathcal{H}\left(D_{1}\right)}$ define equivalent norms, the material coefficients having by assumption (subsection 1.1) the requisite positivity properties.

For any $\omega \in \mathbb{C} \backslash\{0\}$, the differences $\boldsymbol{G}_{\omega}-\boldsymbol{G}_{0}$ and $\boldsymbol{\nabla} \boldsymbol{G}_{\omega}-\boldsymbol{\nabla} \boldsymbol{G}_{0}$ are known to be non-singular at the origin, even for the anisotropic elastodynamic Green's tensor, see e.g., [31, 37]. Therefore, the same 
holds for $\boldsymbol{G}_{\omega}-\boldsymbol{G}_{\mathrm{i}}=\left(\boldsymbol{G}_{\omega}-\boldsymbol{G}_{0}\right)-\left(\boldsymbol{G}_{\mathrm{i}}-\boldsymbol{G}_{0}\right)$, implying that $\boldsymbol{\mathcal { A }}_{\omega}-\boldsymbol{\mathcal { A }}_{\mathrm{i}}$ is compact as an operator from $\boldsymbol{H}^{1}\left(D_{1}\right)$ to $\boldsymbol{H}^{1}\left(D_{1}\right)$. This, combined with Lemma 3.3, shows:

Proposition 3.4. The integral operator $\mathcal{I}-\mathcal{A}_{\omega}: \boldsymbol{H}^{1}\left(D_{1}\right) \rightarrow \boldsymbol{H}^{1}\left(D_{1}\right)$ is Fredholm with index 0.

Remark 3.5. The formulation and proof of Lemma 3.3 adapt and modify [24, Lemma 1] (where transmission problems involving the anisotropic scalar wave equation are considered) and its method of proof to the present context. The coercivity result of [24] rests upon the electromagnetic counterpart of the positive definiteness of $\Delta \mathcal{C}$ (albeit, as stressed therein, alternative lines of reasoning also allow to establish bounded invertibility of $\mathcal{I}-\mathcal{A}_{\mathrm{i}}$ without such restriction).

3.3. Uniqueness. A solution $\boldsymbol{v} \in \boldsymbol{H}_{\mathrm{loc}}^{1}\left(\mathbb{R}^{3}\right)$ to the homogeneous transmission problem (i.e., when $\boldsymbol{u}_{\mathrm{I}}=\mathbf{0}$ ) verifies

$$
\langle\boldsymbol{v}, \overline{\boldsymbol{v}}\rangle_{B_{\varrho}}^{\mathcal{C}}-\omega^{2}(\boldsymbol{v}, \overline{\boldsymbol{v}})_{B_{\varrho}}^{\rho}=\left(\boldsymbol{t}_{0}[\boldsymbol{v}], \overline{\boldsymbol{v}}\right)_{S_{\varrho}},
$$

where $B_{\varrho}$ is the ball $\left\{\boldsymbol{x} \in \mathbb{R}^{3},|\boldsymbol{x}|<\varrho\right\}$ of radius $\varrho$; this stems from taking the inner product of equations (1.4), (1.5) (a) and (1.5) (b) with $\overline{\boldsymbol{v}} \in \boldsymbol{H}^{1}\left(B_{\varrho}\right)$, invoking in each case the first Green identity, and combining the resulting equalities. Taking the imaginary part of the above identity gives

$$
\Im\left(\boldsymbol{t}_{0}[\boldsymbol{v}], \overline{\boldsymbol{v}}\right)_{B_{\varrho}}=0=\Im\left(\boldsymbol{t}_{0}[\overline{\boldsymbol{v}}], \boldsymbol{v}\right)_{B_{\varrho}} .
$$

Hence, $I_{R}(\boldsymbol{v})=0$, as well, with $I_{R}(\boldsymbol{v})$ defined as in Lemma 2.3. The asymptotic form of $I_{R}(\boldsymbol{v})$, given by Lemma 2.3, together with $(-1)^{p} \int_{\hat{S}} \gamma_{p}\left(\widehat{\boldsymbol{\xi}}^{p}\right)\left(2 \pi^{2}\left|\boldsymbol{\nabla} D\left(\boldsymbol{\xi}^{p}\right)\right| \kappa\left(\boldsymbol{\xi}^{p}\right)\right)^{-1}>0$, then implies that $\boldsymbol{I}^{p}(\widehat{\boldsymbol{x}})$. $\boldsymbol{b}^{p}\left(\widehat{\boldsymbol{x}}^{p}\right)=0$ for each $p$. Moreover, any radiating transmission solution is given by the integral representation (2.15), whose far-field asymptotic approximation is of the form (2.7a). Exploiting $\boldsymbol{I}^{p}(\widehat{\boldsymbol{x}}) \cdot \boldsymbol{b}^{p}\left(\widehat{\boldsymbol{x}}^{p}\right)=0$ in the latter implies that:

Lemma 3.6. Radiating solutions to the (homogeneous) transmission problems (1.5) and (1.6) with $\boldsymbol{u}_{I}=\mathbf{0}$, verify $\boldsymbol{v}(\boldsymbol{x})=o\left(|\boldsymbol{x}|^{-1}\right)$. 
The next step consists of showing that $\boldsymbol{v}$ vanishes in $D_{0}$ as a consequence of Lemma 3.6. This requires a Rellich-type lemma, which is not readily available for anisotropic elasticity. We will instead rely on a theorem by Littman [26]. Considering a solution $\boldsymbol{v}$ to the homogeneous transmission problem, which is $C^{\infty}$ in $D_{0}$ by virtue of the integral representation (2.15), let $\widetilde{\boldsymbol{v}}$ denote a $C^{\infty}\left(\mathbb{R}^{3}\right)$ extension of the restriction of $\boldsymbol{v}$ to $\mathbb{R}^{3} \backslash \bar{B}_{R}$, and set $\boldsymbol{f}:=-\boldsymbol{\mathcal { B }}_{0} \widetilde{\boldsymbol{v}}$. The body force density $\boldsymbol{f}$ is a $C^{\infty}\left(\mathbb{R}^{3}\right)$ function with compact support (since, by construction, $\boldsymbol{f}=\mathbf{0}$ is outside of $\left.B_{R}\right)$. Now, the relationship $\boldsymbol{N}(\boldsymbol{\xi}) \cdot \boldsymbol{B}_{0}(\boldsymbol{\xi})=D(\boldsymbol{\xi}) \boldsymbol{I}$ shows that each component of $\widetilde{\boldsymbol{v}}$ satisfies the sixth-order scalar PDE,

$$
D(-\mathrm{i} \nabla) \widetilde{v}_{i}=N_{i j}(-\mathrm{i} \boldsymbol{\nabla}) f_{j}, \quad i=1,2,3 .
$$

Littman's theorem states the following. Consider a partial differential operator $P(-\mathrm{i} \boldsymbol{\nabla})$ (where $P(\boldsymbol{\xi})$, the symbol of the operator, is a polynomial in $\boldsymbol{\xi} \in \mathbb{R}^{3}$ with constant coefficients), such that:

(i) the solution set in $\mathbb{R}^{3}$ of $P(\boldsymbol{\xi})=0$ is the union of a finite number of smooth surfaces,

(ii) $\boldsymbol{\nabla} P(\boldsymbol{\xi}) \neq \mathbf{0}$ on $S$, and

(iii) the Gaussian curvature $\kappa(\boldsymbol{\xi})$ is nonzero at any $\boldsymbol{\xi} \in S$.

If a solution $v$ of the PDE $P(-\mathrm{i} \boldsymbol{\nabla}) v=g$ is such that $v=o\left(|\boldsymbol{x}|^{-1}\right)$ as $|\boldsymbol{x}| \rightarrow \infty$, then $v$ has compact support. Conditions (i), (ii) and (iii) are satisfied here by $P=D$ and $g=N_{i j}(-\mathrm{i} \nabla) f_{j}$ by virtue of $D(\boldsymbol{\xi})$ being the characteristic determinant of $\boldsymbol{B}_{0}(\boldsymbol{\xi})$ and Assumption 1.1. Consequently,

Lemma 3.7. Radiating solutions to the homogeneous transmission problems (1.5) and (1.6) have compact support.

Remark 3.8. The assumptions underpinning Littman's theorem here require the background material properties to have a class I anisotropy.

Since $\boldsymbol{v}_{0}=\mathbf{0}$ in $D_{0} \backslash \operatorname{supp}(\boldsymbol{f})$ and $\boldsymbol{v}_{0}$ solves $\boldsymbol{B}_{0} \boldsymbol{v}_{0}=\mathbf{0}$ in $D_{0}$, which is a homogeneous elliptic PDE with constant coefficients, the unique continuation principle applies, so that $\boldsymbol{v}=\mathbf{0}$ in $D_{0}$. Then, the method used in [7, subsection 5.4] for scalar problems involving orthotropic scatterers allows us to show that $\boldsymbol{v}=\mathbf{0}$ in $D_{1}$ as well. Let $\widetilde{\mathcal{C}}^{1}$ and $\widetilde{\rho}^{1}$ denote continuously differentiable extensions in $B_{R}$ of $\mathcal{C}^{1}$ and $\rho^{1}$ 
that satisfy all relevant physical requirements listed in subsection 1.1, with the ball $B_{R}$ chosen such that $D_{1} \Subset B_{R}$ and with $\widetilde{\mathcal{B}}$ denoting the corresponding elastodynamic operator. Since $\boldsymbol{v}$ verifies: (i) $\boldsymbol{v}_{0}=\mathbf{0}$ in $B_{R} \backslash \overline{D_{1}}$, (ii) the homogeneous transmission conditions $\boldsymbol{v}_{0}=\boldsymbol{v}_{1}$ and $\boldsymbol{t}_{0}\left[\boldsymbol{v}_{0}\right]=\boldsymbol{t}_{1}\left[\boldsymbol{v}_{1}\right]$ on $\Gamma$, and (iii) the field equation $\mathcal{B}_{1} \boldsymbol{v}_{1}=\mathbf{0}$ in $D_{1}$, it satisfies $\widetilde{\mathcal{B}} \boldsymbol{v}=\mathbf{0}$ in $B_{R}$. Elliptic regularity results then imply that $\boldsymbol{v}$ is $C^{1}$ in the interior of $B_{R}$, allowing us to apply the unique continuation principle (see [19, Theorem 17.2.6]), showing that $\boldsymbol{v}=\mathbf{0}$ in $B_{R}$ as a consequence of $\boldsymbol{v}_{0}=\mathbf{0}$ in $D_{0}$. We have thus shown that:

Proposition 3.9. The transmission problems (1.5) and (1.6) have at most one radiating solution.

3.4. Equivalence and well-posedness. Summarizing, we know at this point that:

(1) any radiating transmission solution $\boldsymbol{v}$ is such that $\boldsymbol{u}_{1}=\boldsymbol{u}_{\mathrm{I}}+\boldsymbol{v}_{1}$ solves the integral equation (2.14);

(2) any solution of equation (2.14), together with the integral representation equation (2.15), defines a radiating transmission solution $\boldsymbol{v}=\boldsymbol{u}-\boldsymbol{u}_{\mathrm{I}}$

(3) the integral operator $\mathcal{A}_{\omega}$ of equation (2.14) is Fredholm with index 0 ;

(4) the homogeneous transmission problem has at most one radiating solution.

Moreover, (4) implies uniqueness for the integral equation (2.14) since a non-trivial solution $\boldsymbol{w}$ to $\left(\mathcal{I}-\mathcal{A}_{\omega}\right) \boldsymbol{w}=\mathbf{0}$ would otherwise, by Lemma 3.2, define a radiating solution of the homogeneous transmission problem that is nonzero at least in $D_{1}$, a contradiction. Existence and bounded invertibility for equation (2.14) therefore follows due to (3) and the Fredholm alternative. Our following main result is thus established:

Theorem 3.10. Assume that $D_{1}, \mathcal{C}^{0}, \rho^{0}$ and $\mathcal{C}^{1}, \rho^{1}$ satisfy the assumptions in subsection 1.1 , with $\mathcal{C}^{0}$ additionally constrained by Assumption 1.1, i.e., of class I anisotropy. Then:

(i) the transmission (scattering) problem defined by equations (1.5) and (1.6) for a given incident field $\boldsymbol{u}_{I}$ satisfying $\boldsymbol{B}_{0} \boldsymbol{u}_{I}=\mathbf{0}$ in $\mathbb{R}^{3}$ 
has a unique solution $\boldsymbol{v} \in \boldsymbol{H}_{\mathrm{loc}}^{1}\left(\mathbb{R}^{3}\right)$ that is radiating in the sense of Definition 2.6;

(ii) The integral operator $\mathcal{I}-\mathcal{A}_{\omega}: \boldsymbol{H}^{1}\left(D_{1}\right) \rightarrow \boldsymbol{H}^{1}\left(D_{1}\right)$ is invertible with bounded inverse;

(iii) The unique solution $\boldsymbol{u}_{1} \in \boldsymbol{H}^{1}\left(D_{1}\right)$ of integral equation (2.14) is the restriction of $\boldsymbol{u}$ to $D_{1}$;

(iv) The total field $\boldsymbol{u}_{0}=\boldsymbol{u}_{I}+\boldsymbol{v}_{0}$ outside the inhomogeneity is given in terms of $\boldsymbol{u}_{1}$ by the integral representation formula (2.15).

3.5. General transmission problem. A more general form of the transmission problem consists of seeking displacement fields $\boldsymbol{u}_{1}$ in $D_{1}$ and $\boldsymbol{v}_{0}$ in $D_{0}$ solving

(1) the field equations of linear elastodynamics
(a) $\mathcal{B}_{0} \boldsymbol{v}_{0}=\mathbf{0}$ in $D_{0}$,
(b) $\boldsymbol{B}_{1} \boldsymbol{u}_{1}=\mathbf{0}$ in $D_{1}$,

(2) the transmission conditions
(a) $\boldsymbol{u}_{1}=\boldsymbol{v}_{0}+\boldsymbol{f}$
(b) $\boldsymbol{t}_{1}\left[\boldsymbol{u}_{1}\right]=\boldsymbol{t}_{0}\left[\boldsymbol{v}_{0}\right]+\boldsymbol{g}$ on $\Gamma$,

for given $\boldsymbol{f}, \boldsymbol{g}$, and

(3) the radiation condition $\mathcal{M}\left[\boldsymbol{v}_{0}\right]=\mathbf{0}$.

The scattering problem studied in this article then corresponds to $(\boldsymbol{f}, \boldsymbol{g})=\left(\boldsymbol{u}_{\mathrm{I}}, \boldsymbol{t}_{0}\left[\boldsymbol{u}_{\mathrm{I}}\right]\right)$. Upon adapting the proof of Proposition 2.5 to this case, the above version of the transmission problem leads to an integral representation identity which differs from equation (2.15) by involving layer potentials on $\Gamma$ with densities $\boldsymbol{f}$ and $\boldsymbol{g}$ in addition to the volume potentials. The latter cancel out, as expected, whenever $\boldsymbol{f}$ and $\boldsymbol{g}$ are compatible, i.e., $(\boldsymbol{f}, \boldsymbol{g})=\left(\boldsymbol{u}_{\mathrm{I}}, \boldsymbol{t}_{0}\left[\boldsymbol{u}_{\mathrm{I}}\right]\right)$ for some $\boldsymbol{u}_{\mathrm{I}}$ solving $\mathcal{B}_{0} \boldsymbol{u}_{\mathrm{I}}=\mathbf{0}$.

3.6. Inhomogeneity with piecewise-smooth properties. The solvability result of Theorem 3.10 can be extended to scatterers for which $\mathcal{C}^{1}$ and $\rho^{1}$ are piecewise $C^{1, \alpha}$ in $D_{1}$, assuming that the interfaces between components of $D_{1}$ with smooth properties (a) have only two such components adjacent at any point (e.g., no triple interfacial point), and (b) are separated from $\Gamma$. The unique continuation principle can then be applied stepwise through each interface, while the representation formula of Lemma 2.5 can be shown to satisfy the requisite transmission 
conditions at the new interfaces, and the coercivity result of Lemma 3.3 relies only on $\mathcal{C}^{1}$ and $\rho^{1}$ to be positive and bounded away from zero.

4. Isotropic background material. The foregoing analysis covers the important case of isotropic background materials, which belong to class I. Some features of this case are, however, worth discussing, as (a) there are only two distinct characteristic surfaces, and (b) radiating solutions can be defined using the Kupradze-Sommerfeld radiation conditions, which raises the issue of equivalence with the present constraint $\mathcal{M}[\boldsymbol{v}]=\mathbf{0}$.

An isotropic background material is characterized by an elasticity tensor $\mathcal{C}^{0}$ of the form $\mathcal{C}^{0}=\lambda \boldsymbol{I} \otimes \boldsymbol{I}+2 \mu \mathcal{I}$, where $\lambda$ and $\mu$ are the socalled Lamé constants of the elastic material and $\boldsymbol{I}$ and $\mathcal{I}$ denote the second-order and symmetric fourth-order identity tensors, respectively. The fundamental tensor $\boldsymbol{G}$ is available in closed form $[\mathbf{1 4}, \mathbf{2 5}]$ :

$$
\begin{aligned}
\boldsymbol{G} & =\boldsymbol{G}_{\mathrm{P}}+\boldsymbol{G}_{\mathrm{S}} \\
\boldsymbol{G}_{\mathrm{P}}(\boldsymbol{x}) & =-k_{\mathrm{P}}\left(\mu k_{\mathrm{S}}^{2}\right)^{-1} \nabla \nabla G_{\mathrm{P}}(\boldsymbol{x}), \\
\boldsymbol{G}_{\mathrm{S}}(\boldsymbol{x}) & =\left(\mu k_{\mathrm{S}}\right)^{-1}\left(k_{\mathrm{S}}^{2} \boldsymbol{I}+\nabla \boldsymbol{\nabla}\right) G_{\mathrm{S}}(\boldsymbol{x}),
\end{aligned}
$$

where the functions $G_{\alpha}(\alpha=\mathrm{P}, \mathrm{S})$ are defined by $G_{\alpha}(\boldsymbol{x})=g\left(k_{\alpha}|\boldsymbol{x}|\right)$ with $g(t):=e^{\mathrm{i} t} /(4 \pi t)$, and with $k_{\mathrm{P}}:=\omega\left[\rho^{0} /(\lambda+2 \mu)\right]^{1 / 2}$ and $k_{\mathrm{S}}:=\omega\left(\rho^{0} / \mu\right)^{1 / 2}$ denoting the wavenumbers of compression and shear elastic bulk waves, respectively. Equation (4.1) in fact gives the Helmholtz decomposition of $\boldsymbol{G}$ in terms of its irrotational and solenoidal parts $\boldsymbol{G}_{\mathrm{P}}$ and $\boldsymbol{G}_{\mathrm{S}}$ (since $\boldsymbol{\nabla} \times \boldsymbol{G}_{\mathrm{P}}(\boldsymbol{x})=\mathbf{0}$ and $\operatorname{div} \boldsymbol{G}_{\mathrm{S}}(\boldsymbol{x})=\mathbf{0}$ for any $\left.\boldsymbol{x} \neq \mathbf{0}\right)$. The far-field behavior of $\boldsymbol{G}$ and $\boldsymbol{T}$ is characterized by

$$
\begin{aligned}
\boldsymbol{G}_{\mathrm{P}}(\boldsymbol{x}) & =\frac{k_{\mathrm{P}}}{\lambda+2 \mu} G_{\mathrm{P}}(\boldsymbol{x}) \widehat{\boldsymbol{x}} \otimes \widehat{\boldsymbol{x}}+o\left(|\boldsymbol{x}|^{-1}\right), \\
\boldsymbol{G}_{\mathrm{S}}(\boldsymbol{x}) & =\frac{k_{\mathrm{S}}}{\mu} G_{\mathrm{S}}(\boldsymbol{x})(\boldsymbol{I}-\widehat{\boldsymbol{x}} \otimes \widehat{\boldsymbol{x}})+o\left(|\boldsymbol{x}|^{-1}\right), \\
\boldsymbol{T}_{\mathrm{P}}(\boldsymbol{x}) & =\mathrm{i} k_{\mathrm{P}}^{2} G_{\mathrm{P}}(\boldsymbol{x}) \widehat{\boldsymbol{x}} \otimes \widehat{\boldsymbol{x}}+o\left(|\boldsymbol{x}|^{-1}\right), \\
\boldsymbol{T}_{\mathrm{S}}(\boldsymbol{x}) & =\mathrm{i} k_{\mathrm{S}}^{2} G_{\mathrm{S}}(\boldsymbol{x})(\boldsymbol{I}-\widehat{\boldsymbol{x}} \otimes \widehat{\boldsymbol{x}})+o\left(|\boldsymbol{x}|^{-1}\right) .
\end{aligned}
$$


4.1. Characteristic surfaces. The characteristic equation $D(\boldsymbol{\xi})=0$ takes the form

$$
\mu^{2}(\lambda+2 \mu)\left(|\boldsymbol{\xi}|^{2}-k_{\mathrm{S}}^{2}\right)^{2}\left(|\boldsymbol{\xi}|^{2}-k_{\mathrm{P}}^{2}\right)=0 .
$$

Hence, $S^{1}$ and $S^{2}$ are identical spheres of radius $k_{\mathrm{S}}$ in $\boldsymbol{\xi}$-space while $S^{3}$ is a sphere of radius $k_{\mathrm{P}}$; moreover, $\boldsymbol{b}^{3}(\widehat{\boldsymbol{\xi}})=\widehat{\boldsymbol{\xi}}$. The vectors $\boldsymbol{\xi}^{q}(\widehat{\boldsymbol{x}})$ associated with an observation direction $\widehat{\boldsymbol{x}}$ are $\boldsymbol{\xi}^{1}(\widehat{\boldsymbol{x}})=\boldsymbol{\xi}^{2}(\widehat{\boldsymbol{x}})=k_{\mathrm{S}} \widehat{\boldsymbol{x}}$ and $\boldsymbol{\xi}^{3}(\widehat{\boldsymbol{x}})=k_{\mathrm{P}} \widehat{\boldsymbol{x}}$ and so are, in particular, collinear to $\widehat{\boldsymbol{x}}$. The adjugate matrix $\boldsymbol{N}(\boldsymbol{\xi})$ of $\boldsymbol{B}_{0}(\boldsymbol{\xi})$ is given (having omitted the constant factor $\mu(\lambda+2 \mu))$ by

$$
\boldsymbol{N}(\boldsymbol{\xi})=\left(|\boldsymbol{\xi}|^{2}-k_{\mathrm{P}}^{2}\right) \boldsymbol{I}-\left(|\boldsymbol{\xi}|^{2}-k_{\mathrm{S}}^{2}\right) \widehat{\boldsymbol{\xi}} \otimes \widehat{\boldsymbol{\xi}}
$$

and satisfies $\boldsymbol{B}_{0}(\boldsymbol{\xi}) \cdot \boldsymbol{N}(\boldsymbol{\xi})=\left(|\boldsymbol{\xi}|^{2}-k_{\mathrm{S}}^{2}\right)\left(|\boldsymbol{\xi}|^{2}-k_{\mathrm{P}}^{2}\right) \boldsymbol{I}$. Littman's theorem applies with $\boldsymbol{N}(\boldsymbol{\xi})$ thus defined; alternatively, a Rellich-type lemma is available.

4.2. Radiating character of energy flux. Equations (4.2) yield

$$
\begin{aligned}
{\left[\boldsymbol{G}^{k} \cdot \overline{\boldsymbol{T}}^{k}\right](\boldsymbol{x}) } & =\left[\left(\boldsymbol{e}_{k} \cdot \boldsymbol{G}\right) \cdot\left(\boldsymbol{e}_{k} \cdot \overline{\boldsymbol{T}}\right)\right](\boldsymbol{x}) \\
& =\frac{-\mathrm{i}}{(4 \pi|\boldsymbol{x}|)^{2}}\left[\frac{k_{\mathrm{P}}}{\lambda+2 \mu} \widehat{x}_{k}^{2}+\frac{k_{\mathrm{S}}}{\mu}\left(1-\widehat{x}_{k}^{2}\right)\right]+o\left(|\boldsymbol{x}|^{-2}\right),
\end{aligned}
$$

implying $\Im\left(\boldsymbol{G}^{k}(\boldsymbol{x}) \cdot \overline{\boldsymbol{T}}^{k}(\boldsymbol{x})\right)<0$ for large enough $|\boldsymbol{x}|$. Likewise, the far-field asymptotic behavior of any displacement field of the form $\boldsymbol{w}=\mathcal{V}_{\omega}[\boldsymbol{g}]+\mathcal{W}_{\omega}[\boldsymbol{h}]=\mathcal{V}_{\omega}[\boldsymbol{g}+\operatorname{div} \boldsymbol{h}]-\mathcal{S}_{\omega}[\boldsymbol{h} \cdot \boldsymbol{n}]$ is found from equation (4.2), by straightforward computations to be given by

$$
\begin{aligned}
\boldsymbol{w}(\boldsymbol{x})= & \frac{k_{\mathrm{P}}}{\lambda+2 \mu} G_{\mathrm{P}}(\boldsymbol{x})\left[\boldsymbol{I}_{\mathrm{P}}(\widehat{\boldsymbol{x}}) \cdot \widehat{\boldsymbol{x}}\right] \widehat{\boldsymbol{x}} \\
& +\frac{k_{\mathrm{S}}}{\mu} G_{\mathrm{S}}(\boldsymbol{x})\left(\boldsymbol{I}_{\mathrm{S}}(\widehat{\boldsymbol{x}})-\left[\boldsymbol{I}_{\mathrm{S}}(\widehat{\boldsymbol{x}}) \cdot \widehat{\boldsymbol{x}}\right] \widehat{\boldsymbol{x}}\right)+o\left(|\boldsymbol{x}|^{-1}\right)
\end{aligned}
$$

with

$$
\begin{aligned}
\boldsymbol{I}_{\alpha}(\widehat{\boldsymbol{x}})= & \int_{D_{1}} e^{-\mathrm{i} k_{\alpha} \widehat{\boldsymbol{x}} \cdot \boldsymbol{y}}(\boldsymbol{g}+\operatorname{div} \boldsymbol{h})(\boldsymbol{y}) \mathrm{d} V_{y} \\
& -\int_{\Gamma} e^{-\mathrm{i} k_{\alpha} \widehat{\boldsymbol{x}} \cdot \boldsymbol{y}} \boldsymbol{h}(\boldsymbol{y}) \cdot \boldsymbol{n}(\boldsymbol{y}) \mathrm{d} S_{y} \quad(\alpha=\mathrm{P}, \mathrm{S}),
\end{aligned}
$$


from which we deduce that

$$
\begin{aligned}
\boldsymbol{t}[\overline{\boldsymbol{w}}](\boldsymbol{x}) \cdot \boldsymbol{w}(\boldsymbol{x})= & -\mathrm{i}\left[\frac{k_{\mathrm{P}}}{\lambda+2 \mu}\left|\boldsymbol{I}_{\mathrm{P}}(\widehat{\boldsymbol{x}}) \cdot \widehat{\boldsymbol{x}}\right|^{2}\right. \\
& \left.+\frac{k_{\mathrm{S}}}{\mu}\left(\left|\boldsymbol{I}_{\mathrm{S}}(\widehat{\boldsymbol{x}})\right|^{2}-\left|\boldsymbol{I}_{\mathrm{S}}(\widehat{\boldsymbol{x}}) \cdot \widehat{\boldsymbol{x}}\right|^{2}\right)\right] \frac{1}{(4 \pi|\boldsymbol{x}|)^{2}}+o\left(|\boldsymbol{x}|^{-2}\right),
\end{aligned}
$$

implying $\Im(\boldsymbol{t}[\overline{\boldsymbol{w}}] \cdot \boldsymbol{w})(\boldsymbol{x})<0$ if $|\boldsymbol{x}|$ is large enough. Equations (4.3) and (4.4) are the counterpart of Lemma 2.3 for the isotropic case; they evidence the physically radiating character of $\boldsymbol{G}^{k}$ and of volume potentials, respectively.

4.3. Radiating fields and radiation conditions. It is well known that any displacement $\boldsymbol{v}$ solving $\boldsymbol{B}_{0} \boldsymbol{w}=\mathbf{0}$ in the isotropic case can be additively decomposed into irrotational and solenoidal parts, i.e., $\boldsymbol{v}=\boldsymbol{v}_{\mathrm{P}}+\boldsymbol{v}_{\mathrm{S}}$ with $\operatorname{div} \boldsymbol{v}_{\mathrm{P}}=0$ and $\boldsymbol{\nabla} \times \boldsymbol{v}_{\mathrm{S}}=\mathbf{0}$. Radiating solutions $\boldsymbol{v}$ can then be defined as satisfying the Kupradze-Sommerfeld radiation conditions [25]:

$$
\left(\partial_{r}-\mathrm{i} k_{\mathrm{P}}\right) \boldsymbol{v}_{\mathrm{P}}(\boldsymbol{y})=o\left(|\boldsymbol{y}|^{-1}\right), \quad\left(\partial_{r}-\mathrm{i} k_{\mathrm{S}}\right) \boldsymbol{v}_{\mathrm{S}}(\boldsymbol{y})=o\left(|\boldsymbol{y}|^{-1}\right),
$$

which are known [25, Chapter 3, Theorem 2.9] to be equivalent to

$$
\begin{array}{r}
\boldsymbol{t}_{0}\left[\boldsymbol{v}_{\mathrm{P}}\right](\boldsymbol{y})-\mathrm{i} k_{\mathrm{P}}(\lambda+2 \mu) \boldsymbol{v}_{\mathrm{P}}(\boldsymbol{y})=o\left(|\boldsymbol{y}|^{-1}\right), \\
\boldsymbol{t}_{0}\left[\boldsymbol{v}_{\mathrm{S}}\right](\boldsymbol{y})-\mathrm{i} k_{\mathrm{S}} \mu \boldsymbol{v}_{\mathrm{S}}(\boldsymbol{y})=o\left(|\boldsymbol{y}|^{-1}\right) .
\end{array}
$$

In particular, $\boldsymbol{v}_{\alpha}=\boldsymbol{G}_{\alpha}^{k}(\cdot-\boldsymbol{x})$ verifies (4.6) for $k=1,2,3, \alpha=\mathrm{P}, \mathrm{S}$ and any fixed $\boldsymbol{x} \in \mathbb{R}^{3}$.

Proposition 4.1. Let $\boldsymbol{v}$ solve the transmission problems (1.5) and (1.6), the background medium being isotropic. The Kupradze-Sommerfeld radiation conditions (4.6) are equivalent to the requirement $\mathcal{M}[\boldsymbol{v}]=\mathbf{0}$.

Proof. First, assume that $\boldsymbol{v}$ satisfies the radiation conditions (4.6). It is a known result (see e.g., [25, Chapter 3, subsection 2.4]) that conditions (4.6), together with properties (4.2), ensure

$$
\lim _{A \rightarrow \infty} \int_{S_{A}}\left(\boldsymbol{G}(\cdot-\boldsymbol{x}) \cdot \boldsymbol{t}_{0}[\boldsymbol{u}]-\boldsymbol{T}(\cdot-\boldsymbol{x}) \cdot \boldsymbol{u}\right) \mathrm{d} S=0
$$


i.e., imply $\mathcal{M}[\boldsymbol{v}](\boldsymbol{x})=\boldsymbol{0}$. Conversely, let $\boldsymbol{v}$ solve equations (1.5) and (1.6), and verify $\boldsymbol{\mathcal { M }}[\boldsymbol{v}]=\mathbf{0}$. Then, from (2.15),

$$
\boldsymbol{v}(\boldsymbol{x})=\mathcal{W}_{\omega}\left[\Delta \mathcal{C}: \varepsilon\left[\boldsymbol{u}_{1}\right]\right](\boldsymbol{x})+\omega^{2} \mathcal{V}_{\omega}\left[\Delta \rho \boldsymbol{u}_{1}\right](\boldsymbol{x}) \quad\left(\boldsymbol{x} \in D_{0}\right)\left(\boldsymbol{x} \in D_{0}\right)
$$

Now, using the decomposition $\boldsymbol{G}=\boldsymbol{G}_{\mathrm{P}}+\boldsymbol{G}_{\mathrm{S}}$ in the above potentials, splitting $\boldsymbol{v}$ into $\boldsymbol{v}=\boldsymbol{v}_{\mathrm{P}}+\boldsymbol{v}_{\mathrm{S}}$ accordingly, evaluating the tractions $\boldsymbol{t}_{0}\left[\boldsymbol{v}_{\mathrm{P}}\right]$ and $\boldsymbol{t}_{0}\left[\boldsymbol{v}_{\mathrm{S}}\right]$ by differentiating the potentials under the integral sign and invoking properties (4.2) (b) of $\boldsymbol{G}$, conditions (4.6) are readily found to be verified by $\boldsymbol{v}$.

4.4. Solvability. The foregoing remarks show that Theorem 3.10 applies to the case of isotropic background elasticity.

5. Anisotropic elastostatics. In the case of anisotropic elastostatics the transmission problem is defined by the field equations (1.5) with $\omega=0$, i.e.,

$$
\begin{array}{ll}
\operatorname{div}\left(\mathcal{C}^{0}: \varepsilon\left[\boldsymbol{u}_{\mathrm{I}}+\boldsymbol{v}_{0}\right]\right)=\mathbf{0} & \text { in } D_{0}, \\
\operatorname{div}\left(\mathcal{C}^{1}: \varepsilon\left[\boldsymbol{u}_{\mathrm{I}}+\boldsymbol{v}_{1}\right]\right)=\mathbf{0} & \text { in } D_{1},
\end{array}
$$

and the transmission conditions (1.6), while $\boldsymbol{u}_{\mathrm{I}}$ solves $\operatorname{div}\left(\mathcal{C}^{0}: \varepsilon\left[\boldsymbol{u}_{\mathrm{I}}\right]\right)=$ $\mathbf{0}$ in $\mathbb{R}^{3}$. For instance, solutions for polynomial "incident" fields $\boldsymbol{u}_{\mathrm{I}}$ are often sought for the purposes of, e.g., evaluating elastic moment tensors [1], that are involved in small-inclusion asymptotics or in the estimation of effective properties.

The static fundamental tensor $\boldsymbol{G}_{0}$ is known, e.g., [30, Chapter 1, equation (5.26)], to be given by

$$
\boldsymbol{G}_{0}(\boldsymbol{x})=\frac{1}{8 \pi^{2}|\boldsymbol{x}|} \int_{0}^{2 \pi} \boldsymbol{A}^{-1}(\widehat{\boldsymbol{\xi}}(\phi)) \mathrm{d} \phi
$$

for any anisotropic elasticity tensor $\mathcal{C}^{0}$, where $\phi \mapsto \widehat{\boldsymbol{\xi}}(\phi)$ is an angular parametrization of the unit circle $\widehat{C}:=\{\widehat{\boldsymbol{\xi}} \in \widehat{S}, \widehat{\boldsymbol{\xi}} \cdot \widehat{\boldsymbol{x}}=0\} . \boldsymbol{G}_{0}$ is clearly a homogeneous function with degree -1 in $\boldsymbol{x}$. Unlike in the timeharmonic case, no restriction needs to be put on $\mathcal{C}^{0}$. The counterpart of Lemma 2.5 is the representation identity

$$
\boldsymbol{u}(\boldsymbol{x})=\mathcal{W}_{0}\left[\Delta \mathcal{C}: \varepsilon\left[\boldsymbol{u}_{1}\right]\right](\boldsymbol{x})+\boldsymbol{u}_{\mathrm{I}}(\boldsymbol{x})+\mathcal{M}\left[\boldsymbol{v}_{0}\right](\boldsymbol{x}),
$$


where $\mathcal{M}\left[\boldsymbol{v}_{0}\right]$ and the volume potential $\mathcal{W}_{0}$ are defined in terms of $\boldsymbol{G}_{0}$. The requirement $\mathcal{M}[\boldsymbol{v}]=\mathbf{0}$ is readily found to define fields $\boldsymbol{v}$ of the form $\boldsymbol{v}_{0}=\mathcal{W}_{0}\left[\Delta \mathcal{C}: \varepsilon\left[\boldsymbol{u}_{1}\right]\right.$, which decay as $\boldsymbol{v}(\boldsymbol{x})=O\left(|\boldsymbol{x}|^{-2}\right)$ and $\boldsymbol{t}_{0}[\boldsymbol{v}](\boldsymbol{x})=O\left(|\boldsymbol{x}|^{-3}\right)$ for $|\boldsymbol{x}| \rightarrow \infty$; conversely, this decay implies $\mathcal{M}[\boldsymbol{v}]=\mathbf{0}$. The volume integral equation is

$$
\left(\mathcal{I}-\mathcal{A}_{0}\right) \boldsymbol{u}_{1}(\boldsymbol{x})=\boldsymbol{u}_{\mathrm{I}}(\boldsymbol{x}) \quad\left(\boldsymbol{x} \in D_{1}\right)
$$

with the integral operator $\mathcal{A}_{0}: \boldsymbol{H}^{1}\left(D_{1}\right) \rightarrow \boldsymbol{H}^{1}\left(D_{1}\right)$ defined by $\mathcal{A}_{0}[\boldsymbol{w}](\boldsymbol{x})=\mathcal{W}_{0}[\Delta \mathcal{C}: \boldsymbol{\varepsilon}[\boldsymbol{w}]](\boldsymbol{x})$. Proposition 3.4 applies for $\omega=0$ and $\rho^{1}=\rho^{0}$, and the integral operator $\mathcal{I}-\mathcal{A}_{0}: \boldsymbol{H}^{1}\left(D_{1}\right) \rightarrow \boldsymbol{H}^{1}\left(D_{1}\right)$ is Fredholm with index 0 .

The static counterpart of (3.3) implies that any transmission solution $\boldsymbol{v}$ of the homogeneous problem (for which $\boldsymbol{u}_{\mathrm{I}}=\mathbf{0}$ ) verifies $\langle\boldsymbol{v}, \boldsymbol{v}\rangle_{B_{\varrho}}^{\mathcal{C}}=\left(\boldsymbol{t}_{0}[\boldsymbol{v}], \boldsymbol{v}\right)_{S_{\varrho}}$. Then, due to the above decay conditions, $\lim _{\varrho \rightarrow 0}\left(\boldsymbol{t}_{0}[\boldsymbol{v}], \boldsymbol{v}\right)_{S_{\varrho}}=0=\lim _{\varrho \rightarrow 0}\langle\boldsymbol{v}, \boldsymbol{v}\rangle_{B_{\varrho}}^{\mathcal{C}}$. The latter limit requires $\boldsymbol{v}$ to be a rigid-body motion in $D_{0}$ and $D_{1}$, which must then vanish in both regions due to decay and transmission conditions. Therefore, there is at most one decaying transmission solution. In conclusion,

Theorem 5.1. Assume that $D_{1}, \mathcal{C}^{0}$ and $\mathcal{C}^{1}$ satisfy the assumptions in subsection 1.1. Let $\boldsymbol{u}_{I}$ be a given displacement field satisfying $\operatorname{div}\left(\mathcal{C}^{0}: \varepsilon\left[\boldsymbol{u}_{I}\right]\right)=\mathbf{0}$ in $\mathbb{R}^{3}$. Then:

(i) The elastostatic transmission problem defined by equations (1.6) and (5.1) has a unique decaying solution $\boldsymbol{v}=\boldsymbol{u}-\boldsymbol{u}_{I} \in \boldsymbol{H}_{\mathrm{loc}}^{1}\left(\mathbb{R}^{3}\right)$;

(ii) The integral operator $\mathcal{I}-\mathcal{A}_{0}: \boldsymbol{H}^{1}\left(D_{1}\right) \rightarrow \boldsymbol{H}^{1}\left(D_{1}\right)$ is invertible with bounded inverse;

(iii) The unique solution $\boldsymbol{u}_{1} \in \boldsymbol{H}^{1}\left(D_{1}\right)$ of integral equation (5.2) is the restriction of $\boldsymbol{u}$ to $D_{1}$;

(iv) The total field $\boldsymbol{u}_{0}=\boldsymbol{u}_{I}+\boldsymbol{v}_{0}$ outside the inhomogeneity is given in terms of $\boldsymbol{u}_{1}$ by $\boldsymbol{u}_{0}(\boldsymbol{x})=\boldsymbol{u}_{I}(\boldsymbol{x})+\mathcal{W}_{0}\left[\Delta \mathcal{C}: \varepsilon\left[\boldsymbol{u}_{1}\right]\right](\boldsymbol{x})$. 


\section{Auxiliary proofs.}

\subsection{Proof of Lemma 2.3.}

Proof of part (a). Using the far-field asymptotics (2.4a) and (2.4b), the integral $I_{R, k}$ is given by

$$
I_{R, k}=\sum_{p, q=1}^{3} I_{R, k}^{p q}+o(1),
$$

where $I_{R, k}^{p q}$ are defined, for $p, q \in\{1,2,3\}$, by

$$
I_{R, k}^{p q}=-\mathrm{i}(-1)^{p+q} R^{-1} \int_{R}^{2 R}\left\{\int_{\hat{S}} e^{\mathrm{i} \varrho \hat{\mathbf{x}} \cdot\left(\boldsymbol{\xi}^{p}-\boldsymbol{\xi}^{q}\right)}\left(\boldsymbol{e}_{k} \cdot \boldsymbol{g}^{p q}(\widehat{\boldsymbol{x}}) \cdot \boldsymbol{e}_{k}\right) \mathrm{d} S_{\hat{x}}\right\} \mathrm{d} \varrho,
$$

$$
\boldsymbol{g}^{p q}(\widehat{\boldsymbol{x}})=E\left(\boldsymbol{\xi}^{p}\right) E\left(\boldsymbol{\xi}^{q}\right) \boldsymbol{N}\left(\boldsymbol{\xi}^{p}\right) \cdot \widehat{\boldsymbol{A}}\left(\widehat{\boldsymbol{x}}, \boldsymbol{\xi}^{q}\right) \cdot \boldsymbol{N}\left(\boldsymbol{\xi}^{q}\right)
$$

First, consider $I_{R, k}^{p q}$ for $p \neq q$. In this case, since $\widehat{\boldsymbol{x}} \cdot\left(\boldsymbol{\xi}^{p}-\boldsymbol{\xi}^{q}\right) \neq 0[\mathbf{3 1}]$, the integrand is an oscillatory function of $\varrho$, and a direct calculation yields that $I_{R, k}^{p q}$ vanishes in the limit $R \rightarrow \infty$ :

$$
R^{-1} \int_{R}^{2 R} e^{\mathrm{i} \varrho \hat{\boldsymbol{x}} \cdot\left(\boldsymbol{\xi}^{p}-\boldsymbol{\xi}^{q}\right)} \mathrm{d} \varrho=O\left(R^{-1}\right)=o(1) .
$$

Then, the remaining integrals $I_{R, k}^{p p}$ are given by

$$
I_{R, k}^{p p}=-\mathrm{i} \int_{\hat{S}}\left(\boldsymbol{e}_{k} \cdot \boldsymbol{g}^{p p}(\widehat{\boldsymbol{x}}) \cdot \boldsymbol{e}_{k}\right) \mathrm{d} S_{\hat{x}}+o(1),
$$

with $\boldsymbol{g}^{p p}$ given by

$$
\boldsymbol{g}^{p p}(\widehat{\boldsymbol{x}})=\frac{1}{2}\left[E\left(\boldsymbol{\xi}^{p}\right)\right]^{2} \boldsymbol{N}\left(\boldsymbol{\xi}^{p}\right) \cdot\left(\widehat{\boldsymbol{A}}\left(\widehat{\boldsymbol{x}}, \boldsymbol{\xi}^{p}\right)+\widehat{\boldsymbol{A}}\left(\boldsymbol{\xi}^{p}, \widehat{\boldsymbol{x}}\right)\right) \cdot \boldsymbol{N}\left(\boldsymbol{\xi}^{p}\right),
$$

by virtue of $(6.1 \mathrm{~b})$, the fact that $\boldsymbol{N}\left(\boldsymbol{\xi}^{p}\right)$ is real symmetric, and $\widehat{\boldsymbol{A}}\left(\boldsymbol{\xi}^{p}, \widehat{\boldsymbol{x}}\right)=\widehat{\boldsymbol{A}}\left(\widehat{\boldsymbol{x}}, \boldsymbol{\xi}^{p}\right)^{\mathrm{T}}$. We now evaluate $\boldsymbol{g}^{p p}(\widehat{\boldsymbol{x}})$ by means of a method used in the proof of [31, Lemma 4.1]. We have $\boldsymbol{B}_{0}(\boldsymbol{\xi}) \cdot \boldsymbol{N}(\boldsymbol{\xi})=D(\boldsymbol{\xi}) \boldsymbol{I}$, and therefore, $\boldsymbol{N}(\boldsymbol{\xi}) \cdot \boldsymbol{B}_{0}(\boldsymbol{\xi}) \cdot \boldsymbol{N}(\boldsymbol{\xi})=D(\boldsymbol{\xi}) \boldsymbol{N}(\boldsymbol{\xi})$. Taking the directional derivative with respect to $\boldsymbol{\xi}$ and in the direction $\boldsymbol{\nu}(\boldsymbol{\xi})$ (denoted by the symbol $\left.\partial_{\nu(\xi)}\right)$ on both sides of the latter equality yields

$$
\partial_{\nu(\xi)} \boldsymbol{N}(\boldsymbol{\xi}) \cdot \boldsymbol{B}_{0}(\boldsymbol{\xi}) \cdot \boldsymbol{N}(\boldsymbol{\xi})+\boldsymbol{N}(\boldsymbol{\xi}) \cdot \partial_{\nu(\xi)} \boldsymbol{B}_{0}(\boldsymbol{\xi}) \cdot \boldsymbol{N}(\boldsymbol{\xi})+\boldsymbol{N}(\boldsymbol{\xi})
$$




$$
\cdot \boldsymbol{B}_{0}(\boldsymbol{\xi}) \cdot \partial_{\nu(\xi)} \boldsymbol{N}(\boldsymbol{\xi})=\left(\partial_{\nu(\xi)} D(\boldsymbol{\xi})\right) \boldsymbol{N}(\boldsymbol{\xi})+D(\boldsymbol{\xi}) \partial_{\nu(\xi)} \boldsymbol{N}(\boldsymbol{\xi}) .
$$

For any $\boldsymbol{\xi} \in S^{p}, D(\boldsymbol{\xi})=0$ and $\boldsymbol{B}_{0}(\boldsymbol{\xi}) \cdot \boldsymbol{N}(\boldsymbol{\xi})=\mathbf{0}$, so that

$$
\boldsymbol{N}(\boldsymbol{\xi}) \cdot \partial_{\nu(\xi)} \boldsymbol{B}_{0}(\boldsymbol{\xi}) \cdot \boldsymbol{N}(\boldsymbol{\xi})=\left(\partial_{\nu(\xi)} D(\boldsymbol{\xi})\right) \boldsymbol{N}(\boldsymbol{\xi}), \quad \boldsymbol{\xi} \in S^{p} .
$$

With the additional observation that $\widehat{\boldsymbol{A}}\left(\widehat{\boldsymbol{x}}, \boldsymbol{\xi}^{p}\right)+\widehat{\boldsymbol{A}}\left(\boldsymbol{\xi}^{p}, \widehat{\boldsymbol{x}}\right)=\partial_{\nu(\xi)} \boldsymbol{B}_{0}\left(\boldsymbol{\xi}^{p}\right)$ (stemming from definition (1.11) of $\boldsymbol{B}_{0}(\boldsymbol{\xi})$ and the quadratic and symmetric character of $\boldsymbol{\xi} \mapsto \boldsymbol{A}(\boldsymbol{\xi})$ ), we obtain

$$
\begin{aligned}
& \boldsymbol{N}\left(\boldsymbol{\xi}^{p}\right) \cdot\left(\widehat{\boldsymbol{A}}\left(\widehat{\boldsymbol{x}}, \boldsymbol{\xi}^{p}\right)+\widehat{\boldsymbol{A}}\left(\boldsymbol{\xi}^{p}, \widehat{\boldsymbol{x}}\right)\right) \cdot \boldsymbol{N}\left(\boldsymbol{\xi}^{p}\right) \\
& =\partial_{\nu(\xi)} D\left(\boldsymbol{\xi}^{p}\right) \boldsymbol{N}\left(\boldsymbol{\xi}^{p}\right) \\
& =(-1)^{p}\left|\boldsymbol{\nabla} D\left(\boldsymbol{\xi}^{p}\right)\right| \gamma_{p}\left(\widehat{\boldsymbol{\xi}}^{p}\right) \boldsymbol{b}^{p}\left(\widehat{\boldsymbol{\xi}}^{p}\right) \otimes \boldsymbol{b}^{p}\left(\widehat{\boldsymbol{\xi}}^{p}\right),
\end{aligned}
$$

where the last equality rests on (1.15) and the fact that $\partial_{\nu(\xi)} D\left(\boldsymbol{\xi}^{p}\right)=$ $\boldsymbol{\nabla} D\left(\boldsymbol{\xi}^{p}\right) \cdot \boldsymbol{\nu}\left(\boldsymbol{\xi}^{p}\right)=(-1)^{p}\left|\boldsymbol{\nabla} D\left(\boldsymbol{\xi}^{p}\right)\right|$ (see the comment after Assumption 1.1). As a result, $\boldsymbol{g}^{p p}(\widehat{\boldsymbol{x}})$ is found to be given by

$$
\boldsymbol{g}^{p p}(\widehat{\boldsymbol{x}})=(-1)^{p} \gamma_{p}\left(\widehat{\boldsymbol{\xi}}^{p}\right)\left(2 \pi^{2}\left|\boldsymbol{\nabla} D\left(\boldsymbol{\xi}^{p}\right)\right| \kappa\left(\boldsymbol{\xi}^{p}\right)\right)^{-1} \boldsymbol{b}^{p}\left(\widehat{\boldsymbol{\xi}}^{p}\right) \otimes \boldsymbol{b}^{p}\left(\widehat{\boldsymbol{\xi}}^{p}\right)
$$

Finally,

$$
\begin{aligned}
\Im\left(I_{R, k}\right)= & -\sum_{p=1}^{3}(-1)^{p} \int_{\hat{S}} \gamma_{p}\left(\widehat{\boldsymbol{\xi}}^{p}\right)\left(2 \pi^{2}\left|\boldsymbol{\nabla} D\left(\boldsymbol{\xi}^{p}\right)\right| \kappa\left(\boldsymbol{\xi}^{p}\right)\right)^{-1}\left(\boldsymbol{e}_{k} \cdot \boldsymbol{b}\left(\widehat{\boldsymbol{\xi}}^{p}\right)\right)^{2} \mathrm{~d} S_{\hat{x}} \\
& +O\left(R^{-1}\right),
\end{aligned}
$$

where the $O(1)$ contribution is negative since $(-1)^{p} \gamma_{p}\left(\widehat{\boldsymbol{\xi}}^{p}\right)>0$.

Proof of part (b). Using the far-field asymptotic form of $\boldsymbol{w}$ and $\boldsymbol{t}_{0}[\boldsymbol{w}]$ (Lemma 2.2$), I_{R}(\boldsymbol{w})$ is given by

$$
\begin{aligned}
I_{R}= & \sum_{p, q=1}^{3} I_{R}^{p q}+o(1), \\
I_{R}^{p q}:= & -\mathrm{i}(-1)^{p+q} R^{-1} \int_{R}^{2 R} \\
& \times \int_{\hat{S}} e^{\mathrm{i} \varrho \hat{\mathbf{x}} \cdot\left(\boldsymbol{\xi}^{p}-\boldsymbol{\xi}^{q}\right)}\left(\boldsymbol{I}^{p}(\widehat{\boldsymbol{x}}) \cdot \boldsymbol{g}^{p q}(\widehat{\boldsymbol{x}}) \cdot \overline{\boldsymbol{I}}^{q}(\widehat{\boldsymbol{x}})\right) \mathrm{d} S_{\hat{x}} \mathrm{~d} \varrho, \quad p, q \in\{1,2,3\},
\end{aligned}
$$

with $\boldsymbol{g}^{p q}(\widehat{\boldsymbol{x}})$ given by $(6.1 \mathrm{~b})$. Using the same argument as in the proof of part (a), we have $I_{R}^{p q}=o(1)$ for $p \neq q$. Then, if $p=q$, the limiting 
behavior of $I_{R}^{p p}$ is easily found by using expression (6.2) of $\boldsymbol{g}^{p p}(\widehat{\boldsymbol{x}})$, yielding the previously claimed asymptotic expression (2.8) of $I_{R}(\boldsymbol{w})$. Then, again noting that $(-1)^{p} \gamma_{p}\left(\widehat{\boldsymbol{\xi}}^{p}\right)>0$, we conclude that $\Im\left(I_{R}\right)<0$ for large enough $R$.

Remark 6.1. The integral over $\widehat{S}$ in (6.1a) would be expected to vanish in the limit $\varrho \rightarrow \infty$ by virtue of a stationary phase argument, which would make radial averaging unnecessary. However, finding the critical points of the phase function in (6.1a) and determining whether or not they are degenerate is involved due to the complicated dependence of $\boldsymbol{\xi}^{q}$ on the observational direction $\widehat{\boldsymbol{x}}$, making radial averaging a simpler approach.

6.2. Proof of fast decay of $\mathcal{A}_{\mathrm{i}}[\boldsymbol{v}]$. We begin by showing exponential decay for $|\boldsymbol{x}| \rightarrow \infty$ of $\boldsymbol{G}(\boldsymbol{x})$ if $\omega=\mathrm{i}$. In this case, (2.2) becomes

$$
\boldsymbol{G}(\boldsymbol{x})=\lim _{R \rightarrow+\infty} \frac{1}{(2 \pi)^{3}} \int_{B_{R}} \boldsymbol{F}(\boldsymbol{\xi}) e^{\mathrm{i} \boldsymbol{x} \cdot \boldsymbol{\xi}} \mathrm{d} V(\boldsymbol{\xi})=\mathcal{F}[\boldsymbol{F}](\boldsymbol{x}),
$$

where the matrix-valued function $\boldsymbol{F}$ is defined by $\boldsymbol{F}(\boldsymbol{\xi})=\left[\boldsymbol{A}(\boldsymbol{\xi})+\rho^{0} \boldsymbol{I}\right]^{-1}$ and $\mathcal{F}$ stands for the three-dimensional Fourier transform (the limit $\epsilon \rightarrow 0$ in (2.2) being straightforward since the matrix $\boldsymbol{A}(\boldsymbol{\xi})+\rho^{0} \boldsymbol{I}$ is invertible for any $\boldsymbol{\xi})$. In fact, $\boldsymbol{F} \in C^{\infty}\left(\mathbb{R}^{3}, \mathbb{C}^{3 \times 3}\right) \cap L^{2}\left(\mathbb{R}^{3}, \mathbb{C}^{3 \times 3}\right)$.

Letting $m=\left(m_{1}, m_{2}, m_{3}\right) \in \mathbb{N}^{3}$ denote a multi-index of length $|m|:=m_{1}+m_{2}+m_{3}$, it is easy to show by induction on $|m|$ that $\partial^{m} \boldsymbol{F}=O\left(|\boldsymbol{\xi}|^{-2-|m|}\right.$ ) for $|\boldsymbol{\xi}| \rightarrow \infty$ (where multi-index notation is used); in particular, $\partial^{m} \boldsymbol{F} \in L^{1}\left(\mathbb{R}^{3}, \mathbb{C}^{3 \times 3}\right)$ for $|m| \geq 2$. Choosing $m$ such that $|m|=2, \partial^{m} \boldsymbol{F}$ is a $C^{\infty}$ function that is summable together with all its derivatives; therefore, $\mathcal{F}\left[\partial^{m} \boldsymbol{F}\right](\boldsymbol{x})$ decays faster than any negative power of $|\boldsymbol{x}|$ as $|\boldsymbol{x}| \rightarrow \infty$ by virtue of well-known properties of the Fourier transform in $L^{1}$. Since, in addition, we have $\mathcal{F}\left[\partial^{m} \boldsymbol{F}\right]=\mathrm{i}^{|m|} \boldsymbol{x}^{m} \mathcal{F}[\boldsymbol{F}], \mathcal{F}[\boldsymbol{F}](\boldsymbol{x})$ itself has a fast decay at infinity.

For isotropic materials, the Fourier integral (6.3) can be evaluated analytically in closed form, showing that $\boldsymbol{G}(\boldsymbol{x})$ in fact decays exponentially fast at infinity in this case.

In view of definition (2.13) of $\mathcal{A}_{\omega}$, the fast decay of $\boldsymbol{G}$ clearly carries over to $\mathcal{A}_{\mathrm{i}}[\boldsymbol{v}](\boldsymbol{x})$ as $|\boldsymbol{x}| \rightarrow \infty$, for any displacement $\boldsymbol{v} \in \mathcal{H}\left(D_{1}\right)$ and compact inhomogeneity region $D_{1}$. This, in particular, ensures that 
$\mathcal{A}_{\mathrm{i}}[\boldsymbol{v}] \in \boldsymbol{H}^{1}\left(\mathbb{R}^{3}\right)$, as $\mathcal{A}_{\mathrm{i}}[\boldsymbol{v}] \in \boldsymbol{H}_{\text {loc }}^{1}\left(\mathbb{R}^{3}\right)$ by known general properties of the volume potentials involved.

\section{REFERENCES}

1. H. Ammari and H. Kang, Polarization and moment tensors with applications to inverse problems and effective medium theory, Appl. Math. Sci. 162, SpringerVerlag, New York, 2007.

2. M. Bonnet and G. Delgado, The topological derivative in anisotropic elasticity, Quart. J. Mech. Appl. Math. 66 (2013), 557-586.

3. M. Born and E. Wolf, Principles of optics: Electromagnetic theory of propagation, interference and diffraction of light, Cambridge University Press, Cambridge, 1999.

4. V.T. Buchwald, Elastic waves in anisotropic media, Proc. Roy. Soc. Lond. 253 (1959), 563-580.

5. D.E. Budreck and J.H. Rose, Three-dimensional inverse scattering in anisotropic elastic media, Inverse Prob. 6 (1990), 331-348.

6. R. Burridge, P. Chadwick and A.N. Norris, Fundamental elastodynamic solutions for anisotropic media with ellipsoidal slowness surfaces, Proc. Roy. Soc. Lond. 440 (1993), 655-681.

7. F. Cakoni and D. Colton, Qualitative methods in inverse scattering theory, Springer-Verlag, New York, 2006.

8. W.C. Chew, Waves and fields in inhomogeneous media. IEEE Press, New York, 1995.

9. D. Colton and R. Kress, Inverse acoustic and electromagnetic scattering theory, Springer-Verlag, New York, 1998.

10. M. Costabel, E. Darrigrand and E.H. Koné, Volume and surface integral equations for electromagnetic scattering by a dielectric body, J. Comp. Appl. Math. 234 (2010), 1817-1825.

11. M. Costabel, E. Darrigrand and H. Sakly, The essential spectrum of the volume integral operator in electromagnetic scattering by a homogeneous body, Comp. Rend. Math. 350 (2012), 193-197.

12. M. Costabel and M. Dauge, On representation formulas and radiation conditions, Math. Meth. Appl. Sci. 20 (1997), 133-150.

13. M. Costabel and E.P. Stephan, Integral equations for transmission problems in linear elasticity, J. Int. Equations Appl. 2 (1990), 211-223.

14. A.C. Eringen and E.S. Suhubi, Elastodynamics, Volume II-Linear theory, Academic Press, New York, 1975.

15. D. Gilbarg and N.S. Trudinger, Elliptic partial differential equations of second order, Springer-Verlag, New York, 2001.

16. D. Gintides and K. Kiriaki, Solvability of the integrodifferential equation of Eshelby's equivalent inclusion method, Quart. J. Mech. Appl. Math. 68 (2015), $85-96$. 
17. D. Gridin, Far-field asymptotics of the Green's tensor for a transversely isotropic solid, Proc. Roy. Soc. Lond. 456 (2000), 571-591.

18. P. Hähner, A uniqueness theorem in inverse scattering of elastic waves, IMA J. Appl. Math. 51 (1993), 201-215.

19. L. Hörmander, The analysis of linear partial differential operators, III, Springer-Verlag, New York, 1985.

20. G.C. Hsiao and W.L. Wendland, Boundary integral equations, Springer, New York, 2008.

21. A. Kirsch, An integral equation approach and the interior transmission problem for Maxwell's equations, Inv. Prob. Imag. 1 (2007), 107-127.

22. __ An integral equation for Maxwell's equations in a layered medium with an application to the factorization method, J. Integral Equations Appl. 19 (2007), 333-358.

23. An integral equation for the scattering problem for an anisotropic medium and the factorization method, in Advanced Topics in scattering and biomedical engineering, A. Charalambopoulos, D.I. Fotiadis and D. Polyzos, eds., World Scientific, Singapore, 2008.

24. A. Kirsch and A. Lechleiter, The operator equations of Lippmann-Schwinger type for acoustic and electromagnetic scattering problems in $L^{2}$, Appl. Anal. $\mathbf{8 8}$ (2009), 807-830.

25. V.D. Kupradze, ed., Three-dimensional problems of the mathematical theory of elasticity and thermoelasticity, North Holland, Amsterdam, 1979.

26. W. Littman, Decay at infinity of solutions to partial differential equations with constant coefficients, Trans. Amer. Math. Soc. 123 (1966), 449-459.

27. A. Madyarov and B.B. Guzina, A radiation condition for layered elastic media, J. Elast. 82 (2006), 73-98.

28. P.A. Martin, Acoustic scattering by inhomogeneous obstacles, SIAM J. Appl. Math. 64 (2003), 297-308.

29. W. McLean, Strongly elliptic systems and boundary integral equations, Cambridge University Press, Cambridge, 2000.

30. T. Mura, Micromechanics of defects in solids, Martinus Nijhoff, Dordrecht, 1987.

31. D. Natroshvili, Boundary integral equation method in the steady state oscillation problems for anisotropic bodies, Math. Meth. Appl. Sci. 20 (1997), 95-119.

32. G. Pelekanos, A. Abubakar and P.M. Van den Berg, Contrast source inversion methods in elastodynamics, J. Acoust. Soc. Amer. 114 (2003), 2825-2834.

33. R. Potthast, Electromagnetic scattering from an orthotropic medium, J. Integral Equations Appl. 11 (1999), 197-215.

34. J.M. Richardson, Scattering of elastic waves from symmetric inhomogeneities at low frequencies, Wave Motion 6 (1984), 325-336.

35. T. Touhei, A fast volume integral equation method for elastic wave propagation in a half space, Int. J. Solids Struct. 48 (2011), 3194-3208. 
36. B.R. Vainberg, Principles of radiation, limit absorption and limit amplitude in the general theory of partial differential equations, Russian Math. Surv. 21 (1966), 115-193.

37. C.Y. Wang and J.D. Achenbach, Three-dimensional time-harmonic elastodynamic Green's functions for anisotropic solids, Proc. Roy. Soc. Lond. 449 (1995), 441-458.

38. J.R. Willis, A polarization approach to the scattering of elastic waves-II. Multiple scattering from inclusions, J. Mech. Phys. Solids 28 (1980), 307-327.

39. , Polarization approach to the scattering of elastic waves-I. Scattering by a single inclusion, J. Mech. Phys. Solids 28 (1980), 287-305.

POems (ENSta Paristech, CNRS, inRIA, Université Paris-Saclay), 91120 Palaiseau, France

Email address: mbonnet@ensta.fr 